\title{
A New Algorithm for the Numerical Solution of Diffusion Problems Related to the Smoluchowski Equation
}

\author{
By Bernhard Nickel
}

Abteilung Spektroskopie und Photochemische Kinetik, Max-Planck-Institut für biophysikalische Chemie, Am Fassberg 11, D-37077 Göttingen, Germany

Dedicated to Prof. Dr. Dr. h.c. mult. H. Gg. Wagner on the occasion of his 70th birthday

(Received February 2, 2000; accepted February 8, 2000)

\section{Algorithm / Smoluchowski Equation / Diffusion-Influenced Reactions}

\begin{abstract}
Diffusion-influenced reactions can often be described with simple kinetic models, whose basic features are a spherically symmetric potential, a distance-dependent relative diffusion coefficient, and a distance-dependent first-order rate coefficient. A new algorithm for the solution of the corresponding Smoluchowski equation has been developed. Its peculiarities are: (1) A logarithmic increase of the radius; (2) the systematic use of numerical fundamental solutions $w$ of the Smoluchowski equation; (3) the use of polynomials of up to the $8^{\text {th }}$ degree for the definition of the first and second partial derivatives of $w$ with respect to the radius; (4) successive doubling of the total diffusion time. The power of the algorithm is illustrated by examples. In particular its usefulness for the combination of a short-range potential with a large radial range is demonstrated. Some aspects of the algorithm are explained in the context of one-dimensional diffusion. Diffusion in a harmonic potential (Ornstein-Uhlenbeck process) and in a double-minimum potential is treated in detail. It is shown that a detailed balance will in general not lead to the best approximation of the time-dependence of a distribution.
\end{abstract}

\section{Introduction}

Diffusion-influenced reactions can often be described with simple kinetic models, whose basic common feature is the relative diffusive motion of a particle B in the spherically symmetric potential of a particle A [1-20]. For an ensemble of $\mathrm{A} \cdots \mathrm{B}$ pairs, the average relative motion of $\mathrm{A}$ and $\mathrm{B}$ and the disappearance of $\mathrm{A} \cdots \mathrm{B}$ pairs by a reaction of $\mathrm{A}$ or $\mathrm{B}$ can be described with the Smoluchowski Eq. (1): 


$$
\frac{\partial \rho(r, t)}{\partial t}=\frac{1}{r^{2}} \frac{\partial}{\partial r}\left(r^{2} D(r)\left[\frac{\partial \rho(r, t)}{\partial r}+\frac{1}{k_{\mathrm{B}} T} \frac{\mathrm{d} U(r)}{\mathrm{d} r} \rho(r, t)\right]\right)-k(r) \rho(r, t) .
$$

$\rho$ is a probability density (often normalized for $t=0$ ), $r$ is the distance between the centers of $\mathrm{A}$ and $\mathrm{B}, t$ is the time, $D$ is the distance-dependent relative diffusion coefficient of $\mathrm{A}$ and $\mathrm{B}, k_{\mathrm{B}}$ is the Boltzmann constant, $T$ is the temperature, $U$ is a spherically-symmetric potential, and $k$ is a distancedependent first-order rate coefficient. The partial differential Eq. (1) is to be solved numerically for a given initial condition $\rho(r, 0)$, subject to adequate boundary conditions.

The development of the present algorithm started from a specific problem, which is described in the Appendix. Technically this problem is characterized by the combination of a short-range deep potential with a large radial range. The basic assumption has been that a standard algorithm would not yield sufficiently accurate numerical solutions of problems of this kind within an acceptable computation time. In view of the lot of systematic work on the development of efficient diffusion algorithms $[6-8$, $13-20]$, the publication of a new algorithm requires a justification. In particular it should become evident that the new algorithm incorporates features that are of interest beyond the specific kinetic problem, for whose solution its has been designed. This justification is given in part in the following items and in part in the Discussion in section 4.

Dimension. It has been customary to treat one-dimensional diffusion, circularly symmetric two-dimensional diffusion, and spherically symmetric three-dimensional diffusion formally in the same way by using general formulae, in which the dimension is specified by a dimension variable $d$ (cf. for instance ref. [19, 20]). The present algorithm was originally developed only for spherically symmetric diffusion. Later it turned out that some peculiarities of the algorithm are most easily explained and tested in the context of one-dimensional diffusion, and for this reason one-dimensional diffusion has been also included. Circularly symmetric diffusion has not been taken into account, but its treatment by the present algorithm should offer no difficulties.

Discretization of space coordinate. A short-range potential requires small radial steps $\Delta r$, which are unsuitable for the coverage of large radial ranges $\left(R_{0}, R_{N}\right)$, where $R_{0}$ is the inner radius and $R_{N}$ is the outer radius of the spherical shell, to which the motion of the center of B relative to the center of $\mathrm{A}$ is confined. Different strategies were developed to solve this problem: (a) Different constant values of $\Delta r$ are used in sub-intervals of the total range $\left(R_{0}, R_{N}\right)[6,19]$. (b) The discrete radius is logarithmically increased: $r_{i+1}=r_{i} \times(1+q)$ with $0<q=$ const. $\ll 1$, and $z=\ln r$ is introduced as new independent spatial variable [14]. (c) The independent variable $r$ is transformed by means of an isomorphism onto the finite range 
of a new variable $z(r)$. By a suitable choice of the function $z(r)$, an equidistant discretization of $z$ gives an optimal representation of the potential [17, 18]. The strategy of the present algorithm is in part similar to strategy (b). It will be shown that very large radial ranges can be covered by using a very small value $q_{1}$ in the near zone and a larger value $q_{2}$ in the far zone with a smooth transition from $q_{1}$ to $q_{2}$. In contrast to the strategy (b), the radius $r$ is retained as independent spatial variable.

Partial derivatives. In all diffusion algorithms, the first and the second partial derivatives of the distribution function $\rho$ with respect to $r$ at discrete radii $r_{i}, \rho^{\prime}\left(r_{i}, t\right)$ and $\rho^{\prime \prime}\left(r_{i}, t\right)$, are needed, directly or indirectly. The usual procedures for calculating these partial derivatives are based on finite differencing, which is related to polynomial interpolation. The present procedure employs direct polynomial interpolation [21-23]: The polynomial $Y(r)$ of degree $2 J$, centered at $r_{i}$ and passing through $\rho\left(r_{i-J}, t\right), \ldots, \rho\left(r_{i}, t\right), \ldots$, $\rho\left(r_{i+J}, t\right)$, is calculated and the required partial derivatives are defined by $\rho^{\prime}\left(r_{i}, t\right) \approx Y^{\prime}\left(r_{i}\right)$ and $\rho^{\prime \prime}\left(r_{i}, t\right) \approx Y^{\prime \prime}\left(r_{i}\right)$. With this procedure also the partial derivatives of a numerical $\delta$-function are obtained, which is defined by

$$
w\left(r_{i}, 0 \mid r_{n}\right)=V_{n}^{-1} \delta_{i n},
$$

where $V_{n}$ is the volume of the $n$-th spherical shell, $r_{n}$ is its mean radius, and $\delta_{i n}$ is the Kronecker delta $\left(\delta_{i n}=1\right.$ if $i=n$ and $\delta_{i n}=0$ if $\left.i \neq n\right)$.

Unsuitability of standard algorithms. A standard algorithm for the solution of Eq. (1) can be characterized as follows [24-26]: By using a finite difference procedure, the partial derivatives $\rho^{\prime}\left(r_{i}, t\right)$ and $\rho^{\prime \prime}\left(r_{i}, t\right)$ in Eq. (1) are replaced by linear combinations of $\rho\left(r_{i}, t\right)$ and two or more of its neighboring values $\rho\left(r_{i \pm j}, t\right)$ with $j \geq 1$. Thus a single partial differential equation is replaced by a system of $N$ coupled ordinary differential equations, to which Runge-Kutta methods can be applied [21-26]. It can be easily shown that a standard algorithm of this type is unsuitable for the solution of the present problem [23]. For a pure diffusion problem and in the absence of a potential, the total time range of interest is of the order $t_{\mathrm{tot}} \approx\left(R_{N}-R_{0}\right)^{2} / D$. In the presence of a short-range potential, the radial step $\Delta r=r_{i+1}-r_{i}$ in the vicinity of the greatest slope of the potential is limited by the acceptable ratio of neighboring equilibrium values $\rho_{\text {eq }}$,

$$
\sigma^{-1} \leq \rho_{\mathrm{eq}}\left(r_{i+1}\right) / \rho_{\mathrm{eq}}\left(r_{i}\right) \leq \sigma,
$$

with the value of $\sigma$ depending on the particular algorithm and the desired accuracy of the numerical solution; in general $\sigma$ will be in the range $1<\sigma$ $<2$. If the maximum value of $\Delta r$ is fixed according to Eq. (3), then the maximum time step $\Delta t$ in a Runge-Kutta procedure is $\Delta t \approx(\Delta r)^{2} / D$ (in the case of a $\delta$-function $w\left(r_{i}, 0 \mid r_{n}\right), w\left(r_{n}, \Delta t \mid r_{n}\right)$ must remain positive). Thus the total number of time steps is of the order of $t_{\mathrm{to}} / \Delta t \approx\left(R_{N} / \Delta r\right)^{2}$. The problem described in the Appendix would require $t_{\mathrm{tot}} / \Delta t \geq 4 \times 10^{10}$, which 
means that it cannot be easily solved with a standard method. With the present algorithm problems of this kind can be solved without difficulty. In section 3 an extreme example with $\left(R_{N} / \Delta r\right)^{2} \approx 2.5 \times 10^{17}$ will be presented.

Conservation of total probability. An acceptable numerical algorithm must conserve the total probability in the absence of a reaction. In the case of a numerical $\delta$-function $w\left(r_{i}, 0 \mid r_{n}\right)$ that means with respect to the first time step that the weighted loss of probability density at $r_{n}$ is exactly equal to the sum of the weighted gains of probability density at the neighboring grid points $r_{n \pm j}$ with $j=1,2, \ldots, J$. The present polynomial interpolation yields an exact conservation of total probability in the following special case: One-dimensional diffusion, constant steps $\Delta x$, and a potential defined by a polynomial of a degree $\leq 2 J+1$. In the case of spherically symmetric relative diffusion and logarithmically increasing radii, the total probability is no longer exactly conserved, but the relative error can be made extremely small in the absence of a potential. If $q$ in the radial increment factor $(1+q)$ is small and constant, the relative error is approximately proportional to $q^{2 J+2}$. For instance, with $q=0.02$ and $J=4$, the total probability is virtually exactly conserved within the limits of double-precision calculations. In the presence of a potential, the deviations from the exact probability conservation remain very small, if the ratio $\sigma$ of neighboring equilibrium values $\rho_{\mathrm{eq}}$ is small enough, for instance $\sigma \approx 1.5$ for $J=4$. The required conservation of total probability can be always achieved by a very small correction.

Detailed balance. In the case of reflecting boundaries and in the absence of reactions, the final stationary numerical solution $\rho_{\text {stat }}\left(r_{i}\right)$ should be close to the theoretical equilibrium distribution $\rho_{\mathrm{eq}}\left(r_{i}\right)$. In some algorithms the exact equality $\rho_{\text {stat }}\left(r_{i}\right)=\rho_{\mathrm{eq}}\left(r_{i}\right)$ is implemented by a detailed balance [15, $16,19]$. Its meaning is most easily understood in the case of a Monte-Carlo algorithm $[13,15]$ : In the simplest case of one-dimensional diffusion with equidistant grid points, diffusion is characterized by the frequency $\Omega_{i j}$ of hopping from $x_{i}$ to $x_{j}$. In this case, detailed balance means that the frequencies $\Omega_{i j}$ exactly satisfy the equilibrium condition $\Omega_{i j} \rho_{\mathrm{eq}}\left(r_{i}\right)=\Omega_{j i} \rho_{\mathrm{eq}}\left(r_{j}\right)$. A detailed balance seems to be regarded by some authors $[15,16,19]$ as a necessary condition for a good numerical algorithm. The present algorithm does not satisfy this condition, and for this reason alone it might be considered inferior to other algorithms. However, by the example of one-dimensional diffusion in a harmonic potential (Ornstein-Uhlenbeck process $[13,19,27])$ it will be demonstrated that, by requiring a detailed balance, not the best numerical approximation of the evolution of a distribution function is obtained.

Time propagation. With respect to time propagation, two types of algorithms can be distinguished. In algorithms of the first type, the time step $\Delta t$ is small and constant either during the whole time range of interest or at least during rather large time intervals [20]. During each time step, the 
transfer of probability density is restricted to neighboring grid points. The upper limit of $\Delta t$ is roughly given by the requirement that the transfer of probability density from a grid point $r_{i}$ to its two adjacent grid points must not lead to a negative probability density at $r_{i}$ itself. In algorithms of the second type, the so-called Chebyshev time propagation is applied, which permits large time steps and involves simultaneous changes of $\rho$ in many or even all grid points $[16,19]$. This method is similar to a propagation scheme for the time-dependent Schrödinger equation [28-30]. As far as very long time propagations are of interest, an algorithm of the second type might be the first choice among the known algorithms, for a kinetic problem described like that described in the Appendix. The present algorithm belongs to the first type with respect to the first time step, with the difference that $2 J \geq 2$ instead of 2 neighboring points are involved. With respect to the further time propagation, the present algorithm seems to be new. The basic idea is the systematic use of numerical fundamental solutions $w\left(r_{i}, t \mid\right.$ $\left.r_{n}\right)$, in analogy to the use of analytical fundamental solutions $g\left(r_{i}, t \mid r_{n}\right)$ in the analytical treatment of diffusion problems $[1,2,4,5]$. Let the $N$ fundamental solutions at time $t, w\left(r_{i}, t \mid r_{n}\right)(n=1,2, \ldots, N)$ and the initial condition $\rho\left(r_{i}, 0\right)$ be known. $\rho\left(r_{i}, t\right)$ is then given by

$$
\rho\left(r_{i}, t\right)=\sum_{n=1}^{N}\left[\rho\left(r_{n}, 0\right) \times V_{n}\right] \times w\left(r_{i}, t \mid r_{n}\right) .
$$

Eq. (4) is applied also to the fundamental solutions themselves. By successive doubling of the total diffusion time $t$, each fundamental solution at the time $2 t, w\left(r_{i}, 2 t \mid r_{n}\right)$, can be expressed as the weighted sum of all $N$ fundamental solutions at the time $t, w\left(r_{i}, t \mid r_{l}\right)(l=1,2, \ldots, N)$. Since with this procedure the computation time becomes proportional to the logarithm of the total diffusion time, the computation of $\rho\left(r_{i}, t\right)$ can be always extended to the attainment of the final stationary distribution $\rho_{\text {stat }}\left(r_{i}\right)$ (in the absence of reactions and with reflecting boundaries).

Duration of the first time step. In algorithms of the first type, the total computation time is inversely proportional to the time step $\Delta t$. Hence, in general $\Delta t$ should be as large as possible, and for this reason an algorithm that is of second order with respect to time is preferable to one that is only of first order with respect to time like the present algorithm. However, in connection with successive time-doubling, the limitation to first order is no real disadvantage. For very short times $t$, most elements of $w\left(r_{i}, t \mid r_{n}\right)$ are either equal to zero or can be equated with zero because of their extreme smallness; by limiting all numerical operations to elements of $w\left(r_{i}, t \mid r_{n}\right)$ that are not equal to zero, the computation time for the initial time-doubling cycles becomes very short. Therefore $\Delta t$ can be chosen almost arbitrarily small without appreciably increasing the total computation time. Finally it is to be mentioned that the algorithm is completely stable also with large values of $\Delta t$ like the algorithms of the first type. 
Boundary conditions. The present numerical implementation of reflecting or absorbing boundaries is completely analogous to Smoluchowski's theoretical treatment of boundaries [1, 2, 4, 31]. The first $J$ numerical $\delta$-functions $w\left(r_{i}, O \mid r_{n}\right)(n=1,2, \ldots, J)$ are supplemented by their mirror images $w\left(r_{i}, 0 \mid r_{n}\right)(n=0,-1, \ldots,-J+1)$. In the case of a reflecting boundary, the transfer of probability through the boundary during the first time step is exactly compensated by the inverse transfer of probability from the mirror image. In the case of an absorbing boundary, the same mirrorimage numerical $\delta$-functions are used, but now with a negative sign. Other boundary conditions (radiation, constant concentration) are implemented in a similar way.

Reactions. In a standard algorithm, a reaction (the sink term $k(r)$ in Eq. (1)) is taken into account simultaneously with diffusion. In the present algorithm, a reaction is taken into account by a single, very small change of the fundamental solutions at a suitable diffusion time, which is much shorter than $\left(k_{\max }\right)^{-1}$.

Examples. The efficiency of the present algorithm is demonstrated by five examples with an adequate representation of relative errors: (1) Onedimensional diffusion in a harmonic potential (Ornstein-Uhlenbeck process); (2) one-dimensional diffusion in a double-minimum potential that is defined by a polynomial of the $8^{\text {th }}$ degree; (3) three-dimensional diffusion without potential in a large radial range with constant radial increment factor $(1+q)$ and a reflecting or absorbing inner boundary; (4) three-dimensional diffusion with a short-range potential barrier, a distance-dependent relative diffusion coefficient, a small radial range with a constant radial increment factor $(1+q)$, and reflecting boundaries; (5) three-dimensional diffusion with the same short-range potential barrier and the same distancedependent relative diffusion coefficient and also with reflecting boundaries, but with a very large radial range with a variable radial increment factor $(1+q)$.

The structure of the article is as follows: In section 2, the main features of the algorithm are treated in the context of one-dimensional diffusion. In particular it will be shown that the new algorithm permits the numerical solution of the Ornstein-Uhlenbeck problem with high accuracy. In section 3, three-dimensional diffusion, reactions, and computational aspects of the algorithm are treated. In section 4, the merits and deficiencies of the algorithm are discussed. In section 5 the basic features of the algorithm are summarized.

\section{One-dimensional diffusion}

\subsection{Definition of intervals and boundaries}

Let diffusion be restricted by boundaries at $x=X_{0}$ and $x=X_{N}$ and let the interval $\left(X_{0}, X_{N}\right)$ be divided into $N$ intervals of equal width $h=\left(X_{N}-X_{0}\right) /$ 
$N$. In standard algorithms, the interval boundaries $X_{n}=X_{0}+h n(n=0$, $1, \ldots, N)$ are the grid points. To each inner grid point $X_{n}$ belongs a symmetrical interval $\left(X_{n}-h / 2, X_{n}+h / 2\right)$. The disadvantage of this definition of grid points is that the associated intervals of the boundaries $\left(X_{0}, X_{0}+h /\right.$ $2)$ and $\left(X_{N}-h / 2, X_{N}\right)$, are smaller and unsymmetrical. The ensuing conceptual and mathematical difficulties are avoided by choosing the centers $x_{n}$ of the $N$ intervals as grid points: $x_{n}=X_{0}+h\left(n-\frac{1}{2}\right)$ with $(n=1,2, \ldots, N)$.

\subsection{Initial condition and fundamental solutions}

In the case of one-dimensional diffusion with a constant relative diffusion coefficient and in the absence of a reaction, the one-dimensional analogue of the partial differential Eq. (1) is

$$
\frac{\partial \rho(x, t)}{\partial t}=D\left(\frac{\partial^{2} \rho(x, t)}{\partial x^{2}}+\frac{d u(x)}{d x} \frac{\partial \rho(x, t)}{\partial x}+\frac{d^{2} u(x)}{d x^{2}} \rho(x, t)\right),
$$

where $u(x) \equiv U(x) / k_{\mathrm{B}} T$ is a reduced potential. Let the initial condition $\rho(x, 0)$ be numerically given by a normalized set of $N$ values $\rho\left(x_{n}, 0\right)$ :

$$
\sum_{n=1}^{N} \rho\left(x_{n}, 0\right) h=1 \text {. }
$$

The evolution of $\rho, \rho\left(x_{i}, t\right)$, is completely defined by the evolution of the $N$ fundamental solutions, $w\left(x_{i}, t \mid x_{n}\right)$. For the moment it is sufficient to calculate $w\left(x_{i}, t_{\mathrm{a}} \mid x_{n}\right)$, that is, the result of the first diffusion step of duration $t_{\mathrm{a}} \equiv$ $\Delta t$ on $w\left(x_{i}, 0 \mid x_{n}\right)$. The procedure for calculating $w\left(x_{i}, t_{\mathrm{a}} \mid x_{n}\right)$ is essentially the same as that, which one would apply in the direct numerical solution of the partial differential Eq. (5). For arbitrary $t, \rho(x, t+\Delta t)$ can be calculated from $\rho(r, t)$ by using Euler's method [24]:

$$
\rho(x, t+\Delta t) \approx \rho(x, t)+\frac{\partial \rho(x, t)}{\partial t} \Delta t .
$$

For the calculation of $\partial \rho(x, t) / \partial t$ with Eq. (5), one has to know the first and the second partial derivatives $\rho^{\prime}(x, t) \equiv \partial \rho(x, t) / \partial x$ and $\rho^{\prime \prime}(x, t) \equiv \partial^{2} \rho(x, t) /$ $\partial x^{2}$. If $\rho(x, t)$ is known only for discrete values $x_{n}$ with $x_{n}<x_{n+1}$, one way of calculating $\rho^{\prime}\left(x_{n}, t\right)$ and $\rho^{\prime \prime}\left(x_{n}, t\right)$ is to calculate a suitable differentiable function $Y_{n}(x)$ passing through $\rho\left(x_{n}, t\right)$ and its $2 J$ neighboring points $\rho\left(x_{n \pm 1}\right.$, $t), \rho\left(x_{n \pm 2}, t\right), \ldots, \rho\left(x_{n \pm J}, t\right)$, and having the property $Y_{n}^{\prime}\left(x_{n}\right) \approx \rho^{\prime}\left(x_{n}, t\right)$ and $Y_{n}^{\prime \prime}\left(x_{n}\right) \approx \rho^{\prime \prime}\left(x_{n}, t\right)$ [21-23]. If $Y_{n}(x)$ is a polynomial of the degree $2 J(J=$ $1,2, \ldots)$, then its minimum degree is 4 , if inflection points are to be accounted for. The present algorithm is based on the use of polynomials up to the degree $2 J=8$.

\subsection{Definition of fundamental solutions by polynomials}

Let $\left\{y_{n, n+j}(x)\right\}$ be a set of $2 J+1$ polynomials of the degree $2 J$, centered at $x_{n}$ (first subscript), with the property 


$$
y_{n, n+j}\left(x_{n+i}\right)=\delta_{i j} \quad(-J \leq i \leq J),
$$

where $\delta_{i j}$ is the Kronecker delta. $Y_{n}(x)$ can be written as the weighted sum of these polynomials:

$$
Y_{n}(x)=\sum_{j=-J}^{J} \rho\left(x_{n+j}, t\right) y_{n, n+j}(x) .
$$

Eq. (9) is equivalent to Lagrange's interpolation formula [21-23]. The first and the second partial derivatives of $\rho$ with respect to $x$ at $x_{n}$ are then approximately given by

$$
\begin{aligned}
& \rho^{\prime}\left(x_{n}, t\right) \approx Y_{n}^{\prime}\left(x_{n}\right)=\sum_{j=-J}^{J} \rho\left(x_{n+j}, t\right) y_{n, n+j}^{\prime}\left(x_{n}\right), \\
& \rho^{\prime \prime}\left(x_{n}, t\right) \approx Y_{n}^{\prime \prime}\left(x_{n}\right)=\sum_{j=-J}^{J} \rho\left(x_{n+j}, t\right) y_{n, n+j}^{\prime \prime}\left(x_{n}\right) .
\end{aligned}
$$

The same procedure is applied to the formal calculation of $w^{\prime}\left(x_{i}, 0 \mid x_{n}\right)$ and $w^{\prime \prime}\left(x_{i}, 0 \mid x_{n}\right)$ for $i=n, n \pm 1, \ldots, n \pm J$. The only difference is that for each $i$ the sum of $2 J+1$ terms in Eqs. (10) and (11) reduces to a single term:

$$
\begin{aligned}
& w^{\prime}\left(x_{n+j}, 0 \mid x_{n}\right)=h^{-1} y_{n+j, n}^{\prime}\left(x_{n+j}\right), \\
& w^{\prime \prime}\left(x_{n+j}, 0 \mid x_{n}\right)=h^{-1} y_{n+j, n}^{\prime \prime}\left(x_{n+j}\right) .
\end{aligned}
$$

The equality signs in Eqs. (12) and (13) have the meaning of definitions depending on the degree of the polynomials.

A necessary condition for any diffusion algorithm is the conservation of total probability in the absence of reactions. With respect to the numerical $\delta$-functions $w\left(x_{i}, 0 \mid x_{n}\right)$ that means, the weighted decrease of $w$ in the $n$-th interval must be equal to the weighted gains of $w$ in the $2 J$ neighboring intervals or, in other words, the normalization condition is to be satisfied:

$$
h \sum_{j=-J}^{J} w\left(x_{n+j}, t_{\mathrm{a}} \mid x_{n}\right)=1 .
$$

As a corollary of the definition of $w^{\prime}\left(x_{i}, 0 \mid x_{n}\right)$ and $w^{\prime \prime}\left(x_{i}, 0 \mid x_{n}\right)$ by Eqs. (12) and (13), the condition of probability conservation (14) is exactly satisfied, firstly, for free diffusion for arbitrary $J$ and, secondly, for diffusion in a potential that is given by a polynomial up to the degree $2 J+1$.

It is advantageous to prove the correctness of the first preceding statement first for $J=2$ (the simplest nontrivial case). For the formal calculation of the partial derivatives $w^{\prime}\left(x_{i}, 0 \mid x_{n}\right)$ and $w^{\prime \prime}\left(x_{i}, 0 \mid x_{n}\right)$, a polynomial of the $4^{\text {th }}$ degree, centered at $x_{i}$, is calculated. The following five polynomials are needed:

$$
\begin{array}{r}
y_{n, n}(x)=+\left(4 h^{4}\right)^{-1}\left(x-x_{n}+2 h\right)\left(x-x_{n}+h\right)\left(x-x_{n}-h\right) \\
\left(x-x_{n}-2 h\right) \\
y_{n+1, n}(x)=-\left(6 h^{4}\right)^{-1}\left(x-x_{n}+h\right)\left(x-x_{n}-h\right)\left(x-x_{n}-2 h\right) \\
\left(x-x_{n}-3 h\right)
\end{array}
$$




$$
\begin{array}{r}
y_{n-1, n}(x)=-\left(6 h^{4}\right)^{-1}\left(x-x_{n}+3 h\right)\left(x-x_{n}+2 h\right) \\
\left(x-x_{n}+h\right) \\
\left(x-x_{n}-h\right) \\
y_{n+2, n}(x)=+\left(24 h^{4}\right)^{-1}\left(x-x_{n}-h\right)\left(x-x_{n}-2 h\right)\left(x-x_{n}-3 h\right) \\
\left(x-x_{n}-4 h\right) \\
y_{n-2, n}(x)=+\left(24 h^{4}\right)^{-1}\left(x-x_{n}+4 h\right)\left(x-x_{n}+3 h\right)\left(x-x_{n}+2 h\right) \\
\left(x-x_{n}+h\right) .
\end{array}
$$

The first and second derivatives of these polynomials at their respective centers $x_{n+j}$ are:

$$
\begin{aligned}
& y_{n, n}^{\prime}\left(x_{n}\right)=0 \\
& y_{n, n}^{\prime \prime}\left(x_{n}\right)=-\frac{5}{2 h^{2}} \\
& y_{n \pm 1, n}^{\prime}\left(x_{n} \pm h\right)=\mp \frac{2}{3 h} \\
& y_{n \pm 1, n}^{\prime \prime}\left(x_{n} \pm h\right)=+\frac{4}{3 h^{2}} \\
& y_{n \pm 2, n}^{\prime}\left(x_{n} \pm 2 h\right)= \pm \frac{1}{12 h} \\
& y_{n \pm 2, n}^{\prime \prime}\left(x_{n} \pm 2 h\right)=-\frac{1}{12 h^{2}} .
\end{aligned}
$$

The probability is exactly conserved for arbitrary $t_{\mathrm{a}}$,

$$
\left(D h t_{\mathrm{a}}\right) \times \sum_{j=-J}^{J} y_{n+j, n}^{\prime \prime}\left(x_{n}+h j\right)=0,
$$

with $J=2$ in the present case.

The proof of the validity of Eq. (23) for arbitrary $J \geq 1$ runs as follows. In the special case of $\rho\left(x_{n+j}, 0\right)=1$ for $-J \leq j \leq J$, the analogues of Eqs. (9)-(11) are

$$
\begin{aligned}
& Y_{n}\left(x_{n}\right)=\sum_{j=-J}^{J} y_{n, n+j}\left(x_{n}\right)=1, \\
& Y_{n}^{\prime}\left(x_{n}\right)=\sum_{j=-J}^{J} y_{n, n+j}^{\prime}\left(x_{n}\right)=0, \\
& Y_{n}^{\prime \prime}\left(x_{n}\right)=\sum_{j=-J}^{J} y_{n, n+j}^{\prime \prime}\left(x_{n}\right)=0 .
\end{aligned}
$$

The terms of the sum in Eq. (24b) can be written in a different form:

$$
y_{n, n+j}^{\prime \prime}\left(x_{n}\right)=y_{n-j, n}^{\prime \prime}\left(x_{n}-h j\right) .
$$


Table 1. First and second derivatives of the polynomials $y_{n+j, n}(x)$ of the degree $2 J$ at their centers $x_{n}+j h(-J \leq j \leq J)$. If the terms in the rows 3 to 6 are multiplied by 2 , the sum of the terms in each column is equal to zero.

\begin{tabular}{|c|c|c|c|c|}
\hline$J$ & 1 & 2 & 3 & 4 \\
\hline \multirow{2}{*}{$y_{n, n}^{\prime \prime}\left(x_{n}\right)$} & $-\frac{2}{-}$ & -5 & -49 & 205 \\
\hline & $\overline{h^{2}}$ & $-\overline{2 h^{2}}$ & $\overline{18 h^{2}}$ & $\overline{72 h^{2}}$ \\
\hline \multirow{2}{*}{$y_{n \pm 1, n}^{\prime \prime}\left(x_{n} \pm h\right)$} & +1 & $\begin{array}{r}4 \\
\end{array}$ & +3 & $\begin{array}{r}8 \\
\end{array}$ \\
\hline & $\overline{h^{2}}$ & $\overline{3 h^{2}}$ & $\overline{2 h^{2}}$ & $\overline{5 h^{2}}$ \\
\hline \multirow{2}{*}{$y_{n \pm 2, n}^{\prime \prime}\left(x_{n} \pm 2 h\right)$} & & -1 & -3 & -1 \\
\hline & & $\overline{12 h^{2}}$ & $\overline{20 h^{2}}$ & $\overline{5 h^{2}}$ \\
\hline \multirow{2}{*}{$y_{n \pm 3, n}^{\prime \prime}\left(x_{n} \pm 3 h\right)$} & & & +1 & 8 \\
\hline & & & $\overline{90 h^{2}}$ & $\overline{9 \cdot 35 h^{2}}$ \\
\hline \multirow{2}{*}{$y_{n \pm 4, n}^{\prime \prime}\left(x_{n} \pm 4 h\right)$} & & & & -1 \\
\hline & & & & $16 \cdot 35 h^{2}$ \\
\hline$y_{n, n}^{\prime}\left(x_{n}\right)$ & 0 & 0 & 0 & 0 \\
\hline \multirow{2}{*}{$y_{n \pm 1, n}^{\prime}\left(x_{n} \pm h\right)$} & $=1$ & $\mp \underline{2}$ & $\mp \frac{3}{2}$ & -4 \\
\hline & $+\overline{2 h}$ & $+\overline{3 h}$ & $+\overline{4 h}$ & $+\overline{5 h}$ \\
\hline \multirow{2}{*}{$y_{n \pm 2, n}^{\prime}\left(x_{n} \pm 2 h\right)$} & & \pm 1 & \pm 3 & $+\frac{1}{-}$ \\
\hline & & $12 h$ & $20 h$ & $5 h$ \\
\hline \multirow{2}{*}{$y_{n \pm 3, n}^{\prime}\left(x_{n} \pm 3 h\right)$} & & & $\mp 1$ & $\mp 4$ \\
\hline & & & $\overline{60 h}$ & $+\overline{105 h}$ \\
\hline \multirow[t]{2}{*}{$y_{n \pm 4, n}^{\prime}\left(x_{n} \pm 4 h\right)$} & & & & $\pm \frac{1}{}$ \\
\hline & & & & $280 h$ \\
\hline
\end{tabular}

That means, the set of terms in Eq. (24b) is identical with that in Eq. (23); only the order of the terms in one sum is inverted relative to the order of the terms in the other sum. In Table 1 all terms $y_{n+j, n}^{\prime}\left(x_{n}+h j\right)$ and $y_{n+j, n}^{\prime \prime}\left(x_{n}+h j\right)$ are listed for $J=1,2,3,4$.

The total probability is also exactly conserved, if the reduced potential $u(x)$ is given by a polynomial of the degree $m \leq 2 J+1$ and $1 \leq J \leq 4$ :

$$
\begin{aligned}
& u\left(x_{n}+h j\right)=\sum_{k=0}^{m} \alpha_{k}\left(x_{n}+h j\right)^{k}, \\
& u^{\prime}\left(x_{n}+h j\right)=\sum_{k=1}^{m} k \alpha_{k}\left(x_{n}+h j\right)^{k-1}, \\
& \left.u^{\prime \prime}\left(x_{n}+h j\right)=\sum_{k=2}^{m} k-1\right) \alpha_{k}\left(x_{n}+h j\right)^{k-2} .
\end{aligned}
$$

The probability conservation is exact, because for each term of the polynomial $u(x)$ the following relation holds: 


$$
\begin{array}{r}
k(k-1) x_{n}^{k-2} y_{n, n}\left(x_{n}\right)+\sum_{j=-J}^{J} k\left(x_{n}+h j\right)^{k-1} y_{n+j, n}^{\prime}\left(x_{n}+h j\right)=0 \\
(0 \leq k \leq 2 J+1) .
\end{array}
$$

The correctness of Eq. (27) has been proved for $J=1,2,3$, 4. Eq. (27) probably holds for arbitrary $J \geq 1$, but no serious attempt has been made by the author to prove its validity for $J>4$.

\subsection{Fundamental solutions for the first diffusion step}

For the calculation of the fundamental solutions $w\left(x_{n+j}, t_{\mathrm{a}} \mid x_{n}\right)$ with $j=0$, $\pm 1, \ldots, \pm J$ with Eq. (7), the quantities

$$
H_{n, j} \equiv h\left[\partial w\left(x, t \mid x_{n}\right) / \partial t\right]_{x=x_{n+j}, t=0}
$$

are needed, which are according to Eqs. (5), (12) and (13):

$$
H_{n, j}=D\left[y_{n+j, n}^{\prime \prime}\left(x_{n+j}\right)+u^{\prime}\left(x_{n+j}\right) y_{n+j, n}^{\prime}\left(x_{n+j}\right)+u^{\prime \prime}\left(x_{n+j}\right) y_{n+j, n}\left(x_{n+j}\right)\right] .
$$

For calculating the result of the first diffusion step, it is advantageous to define the changes $b_{n, j}$ of $w\left(x_{n+j}, 0 \mid x_{n}\right)$ relative to $w\left(x_{n}, 0 \mid x_{n}\right)$ :

$$
\begin{aligned}
& b_{n, 0}=1+H_{n, 0} t_{\mathrm{a}}, \\
& b_{n, j}=H_{n, j} t_{\mathrm{a}}(j= \pm 1, \pm 2, \ldots, \pm J) .
\end{aligned}
$$

A practical upper limit of $t_{\mathrm{a}}$ is obtained by requiring $0.5 \leq b_{n, 0}<1$ or $0<\left|H_{n, 0} t_{\mathrm{a}}\right| \leq 0.5$. If boundary conditions are neglected, then the effect of the first diffusion step on $w\left(x_{i}, 0 \mid x_{n}\right)$ is

$$
\begin{aligned}
& w\left(x_{n+j}, t_{\mathrm{a}} \mid x_{n}\right)=w\left(x_{n}, 0 \mid x_{n}\right) \times b_{n, j} \text { for } j=0, \pm 1, \ldots, \pm J, \\
& w\left(x_{n+j}, t_{\mathrm{a}} \mid x_{n}\right)=0 \text { for }|j|>J .
\end{aligned}
$$

The quantities $b_{n, j}$ contain all the information that is needed for calculating the evolution of the fundamental solutions in the absence of reactions.

\subsection{Boundary conditions}

Two boundaries are of interest: A left boundary at $X_{0}$ and a right boundary at $X_{N}$. In the following, first the two limiting cases of a completely reflecting boundary and a completely absorbing boundary are treated. The left reflecting boundary is defined by [4]

$$
\begin{aligned}
& \left(\frac{\partial \rho(x, t)}{\partial x}\right)_{x=x_{0}}=0, \\
& \left(\frac{d u(x)}{d x}\right)_{x=x_{0}}=0,
\end{aligned}
$$

and the left absorbing boundary is defined by

$$
\rho\left(X_{0}, t\right)=0 \text { for } t>0 .
$$


These boundary conditions are numerically implemented as follows. First, $2 J$ additional intervals are defined with indices $n=0,-1, \ldots,-(2 J-1)$ and center values $x_{n}=x_{1}+h(n-1)$. Second, the $J$ additional sets of quantities $H_{n, j}, b_{n, j}(n=0,-1, \ldots,-J+1)$ are calculated. Third, in the case of a reflecting left boundary, the loss of probability density in the intervals $1,2, \ldots, J$ due to diffusion from right to left through the left boundary is exactly balanced by the gain of probability density due to diffusion from left to right through the left boundary from the intervals $0,-1, \ldots$, $-(J-1)$, which are symmetrical to the intervals $1,2, \ldots, J$ with respect to the reflecting boundary at $X_{0}$. The following equations refer to the case $J=2$ :

$$
\begin{aligned}
& b_{1,-1}+b_{1,-2}=c_{0}\left(b_{0,+1}+b_{0,+2}\right), \\
& b_{2,-2}=c_{-1} b_{-1,+2} .
\end{aligned}
$$

The factors $c_{0}$ and $c_{-1}$ are equal to unity in the absence of a potential and close to unity in the presence of a potential. The final values of $b_{1, j}$ and $b_{2, j}$, referring to a particular boundary condition $(\mathrm{BC})$, are

$$
\begin{aligned}
& \left(b_{1,0}\right)_{\mathrm{BC}}=b_{1,0}+b_{0,+1} \times c_{0} \times c_{\mathrm{BC}}, \\
& \left(b_{1,+1}\right)_{\mathrm{BC}}=b_{1,+1}+b_{0,+2} \times c_{0} \times c_{\mathrm{BC}}, \\
& \left(b_{2,-1}\right)_{\mathrm{BC}}=b_{2,-1}+b_{-1,+2} \times c_{-1} \times c_{\mathrm{BC}}, \\
& \left(b_{1,-1}\right)_{\mathrm{BC}}=\left(b_{1,-2}\right)_{\mathrm{BC}}=\left(b_{2,-2}\right)_{\mathrm{BC}}=0 .
\end{aligned}
$$

The factor $c_{\mathrm{BC}}$ specifies the particular boundary condition: $c_{\mathrm{BC}}$ is equal to +1 for a reflecting boundary and equal to -1 for an absorbing boundary. The present implementation of the two boundary conditions is the complete analogue of the analytical procedure (cf. refs. [1, 2, 4, 31]). A reflecting or absorbing right boundary is implemented in the same way by defining the corresponding quantities $\left(b_{N, 0}\right)_{\mathrm{BC}},\left(b_{N,-1}\right)_{\mathrm{BC}},\left(b_{N-1,+1}\right)_{\mathrm{BC}}$, and $\left(b_{N,+1}\right)_{\mathrm{BC}}=$ $\left(b_{N,+2}\right)_{\mathrm{BC}}=\left(b_{N-1,+2}\right)_{\mathrm{BC}}=0$. In the following the subscript "BC" will be omitted, and it will be always assumed that the quantities $b_{1, j}, b_{2, j}$ and $b_{N, j}, b_{N-1, j}$ are defined in accord with the chosen boundary condition.

The extension of the definition of the boundary conditions to arbitrary $J \geq 1$ is straightforward. With these redefinitions of the quantities $b_{1, j}, b_{2, j}$, $\ldots, b_{J, j}$ and $b_{N, j}, b_{N-1, j}, \ldots, b_{N-J+1, j}$, Eq. (32) can be applied to the whole range $1 \leq n \leq N$, if the range of the index $j$ is appropriately restricted in the vicinity of a boundary:

$$
\begin{aligned}
& w\left(x_{n+j}, t_{\mathrm{a}} \mid x_{n}\right)=w\left(x_{n}, 0 \mid x_{n}\right) \times b_{n, j} \text { for } j_{1} \leq j \leq j_{2}, \\
& w\left(x_{n+j}, t_{\mathrm{a}} \mid x_{n}\right)=0 \text { for } j<j_{1} \text { or } j>j_{2},
\end{aligned}
$$

where

$$
\begin{aligned}
& j_{1}=-J \text { and } j_{2}=+J \text { for } J+1 \leq n \leq N-J \\
& j_{1}=1-n \text { and } j_{2}=+J \text { for } 1 \leq n \leq J \\
& j_{1}=-J \text { and } j_{2}=N-n \text { for } N-J+1 \leq n \leq J .
\end{aligned}
$$


Two other boundary conditions are of general interest: A radiation boundary can be defined by a linear combination of a reflecting boundary with an absorbing boundary. In practice that means, the factor $c_{\mathrm{BC}}$ in Eqs. (39)(41) may have arbitrary values in the interval $-1 \leq c_{\mathrm{BC}} \leq+1$. A constantconcentration boundary can be implemented as follows. In contrast to the analytical definition, $\rho$ or $w$ is kept constant not at $X_{0}$ or $X_{N}$ but at $x_{1}$ or $x_{N}$. The definition of the quantities $H_{n, j}$ is the same as before. In the special case $J=2$, the condition $w\left(x_{1}, t_{\mathrm{a}} \mid x_{n}\right)=w\left(x_{1}, 0 \mid x_{n}\right)$ is satisfied, if the Eqs. (37) and (38) are replaced by the equations

$$
\begin{aligned}
& b_{1,0}+s_{1,0} b_{0,+1}=1, \\
& b_{2,-1}+s_{2,-1} b_{0,+1}=0, \\
& b_{3,-2}+s_{3,-2} b_{-1,+2}=0 .
\end{aligned}
$$

Thus the new definitions of the quantities $b_{n, j}$ for $n=1,2,3$ are:

$$
\begin{aligned}
& \left(b_{1,0}\right)_{\mathrm{BC}}=b_{1,0}+b_{0,+1} \times s_{1,0}, \\
& \left(b_{1,+1}\right)_{\mathrm{BC}}=b_{1,+1}+b_{0,+2} \times s_{1,0}, \\
& \left(b_{2,-1}\right)_{\mathrm{BC}}=b_{2,-1}+b_{0,+1} \times s_{2,-1}=0, \\
& \left(b_{2,0}\right)_{\mathrm{BC}}=b_{2,0}+b_{0,+2} \times s_{2,-1}, \\
& \left(b_{3,-2}\right)_{\mathrm{BC}}=b_{3,-2}+b_{-1,+2} \times s_{3,-2}, \\
& \left(b_{1,-1}\right)_{\mathrm{BC}}=\left(b_{1,-2}\right)_{\mathrm{BC}}=\left(b_{2,-2}\right)_{\mathrm{BC}}=0 .
\end{aligned}
$$

The extension of this implementation of a constant-concentration boundary to arbitrary $J$ offers no difficulties.

\subsection{The second and subsequent diffusion steps: \\ Successive time doubling}

The fundamental solutions $w\left(x_{i}, t_{\mathrm{a}} m \mid x_{n}\right)$ and arbitrary distribution functions $\rho\left(x_{i}, t_{\mathrm{a}} m\right)$ with $m \geq 2$ can be calculated with one of three different procedures:

Procedure $(I) . w\left(x_{i}, t_{\mathrm{a}} m \mid x_{n}\right)$ or $\rho\left(x_{i}, t_{\mathrm{a}} m\right)$ is calculated by repeatedly using the quantities $b_{l, j}$ :

$$
w\left(x_{i}, t_{\mathrm{a}} m \mid x_{n}\right)=\sum_{j=j_{1}}^{j_{2}} w\left(x_{i+j}, t_{\mathrm{a}}(m-1) \mid x_{n}\right) \times b_{i+j,-j} .
$$

Procedure (I) corresponds to standard procedures. It is the fastest one if only a single fundamental solution is needed and the number of diffusion steps is not extremely large.

Procedure (II). For very long diffusion times it is advantageous, to calculate $w\left(x_{i}, t \mid x_{n}\right)$ not by successive application of the same time step $t_{\mathrm{a}}$, but by successive doubling of the time, and by defining each fundamental solution at the time $t_{m+1}=t_{\mathrm{a}} 2^{m}$ as a linear combination of all $\mathrm{N}$ fundamental solutions at the time $t_{m}=t_{\mathrm{a}} 2^{m-1}$ : 


$$
w\left(x_{i}, t_{\mathrm{a}} \times 2^{m} \mid x_{n}\right)=\sum_{l=1}^{N}\left[w\left(x_{i}, t_{\mathrm{a}} \times 2^{m-1} \mid x_{n}\right) \times h\right] \times w\left(x_{i}, t_{\mathrm{a}} \times 2^{m-1} \mid x_{l}\right) .
$$

The term in brackets is the probability of finding a particle at the time $t_{m}=$ $t_{\mathrm{a}} \times 2^{m-1}$ in the $l$-th interval, if it has been in the $n$-th interval at time $t_{0}=0$ with unit probability. In the following examples always procedure (II) was used.

Procedure (III). In typical applications, the evolution of $\rho, \rho\left(x_{i}, t_{\mathrm{c}} v\right)$, is of interest, where the constant time step $t_{\mathrm{c}}$ is much longer than the first time step $t_{\mathrm{a}}$. The calculation of $\rho\left(x_{i}, t_{\mathrm{c}} v\right)$ by procedure (III) consists of two parts. First, the $N$ fundamental solutions $w\left(x_{i}, t_{\mathrm{c}} \mid x_{n}\right)$ are calculated with procedure (II). Second, $\rho\left(x_{i}, t_{\mathrm{c}} v \mid x_{n}\right)$ with $v=1,2, \ldots$ is calculated by repeatedly using the fundamental solutions $w\left(x_{i}, t_{\mathrm{c}} \mid x_{n}\right)$ :

$$
\rho\left(x_{i}, t_{\mathrm{c}} v\right)=\sum_{l=1}^{N}\left[\rho\left(x_{l}, t_{\mathrm{c}}(v-1)\right) \times h\right] \times w\left(x_{i}, t_{\mathrm{c}} \mid x_{l}\right) .
$$

\subsection{Example 1: Diffusion in a harmonic potential (Ornstein-Uhlenbeck process)}

For one-dimensional diffusion in a harmonic potential $u=\alpha x^{2}$ the analytical solution of Eq. (5) is known [13, 19, 27]:

$$
g\left(x, t \mid x_{n}\right)=\frac{\exp \left(-\alpha\left[\left(x-x_{n} \exp (-2 \alpha D t)\right]^{2}[1-\exp (-4 \alpha D T)]^{-1}\right)\right.}{\sqrt{\pi[1-\exp (-4 \alpha D t)] / \alpha}} .
$$

The following computations were performed with $\alpha=4 \mathrm{~nm}^{-2}, x_{n}=8 \mathrm{~nm}$, $D=6 \times 10^{9} \mathrm{~nm}^{2} \mathrm{~s}^{-1}, h=0.01 \mathrm{~nm}, X_{0}=-5.005 \mathrm{~nm}, X_{N}=9.005 \mathrm{~nm}, N=$ 1401, and reflecting boundaries at $X_{0}$ and $X_{N}$. The initial speed of computation was strongly enhanced by equating all extremely small values of $w\left(x, t \mid x_{n}\right)$ with zero and by limiting all summations to terms that differed from zero. The smallest equilibrium value was $g_{\text {eq }}\left(x_{N}\right) \approx 2.19 \times 10^{-141} \mathrm{~nm}^{-1}$. After each time-doubling cycle, $w\left(x_{i}, t \mid x_{n}\right)$ was equated with zero if $\left|w\left(x_{i}, t \mid x_{n}\right)\right|$ was smaller than $10^{-150} \mathrm{~nm}^{-1}$.

In the steady state, $g_{\mathrm{eq}}(x) \equiv g\left(x, \infty \mid x_{n}\right)$ is a Gaussian centered at $x=0$. $g_{\text {eq }}(x)$ is shown in Fig. 1a on a logarithmic scale. The stationary numerical solutions $w_{\text {stat }}(x) \equiv w\left(x, t_{\text {stat }} \mid x_{n}\right)$ obtained with $J=1,2,3,4$ are compared with the exact solution $g_{\text {eq }}(x)\left(t_{\text {stat }}\right.$ is the time at which all $N$ fundamental functions are equal within a specified accuracy). The relative deviation $F$ of $w$ from $g$ is defined by

$$
F=(w / g)-1 .
$$

Since $F$ changes sign and $|F|$ extends over 10 orders of magnitude and, the following quantity $z$ is used for the graphical representation of relative deviations: 


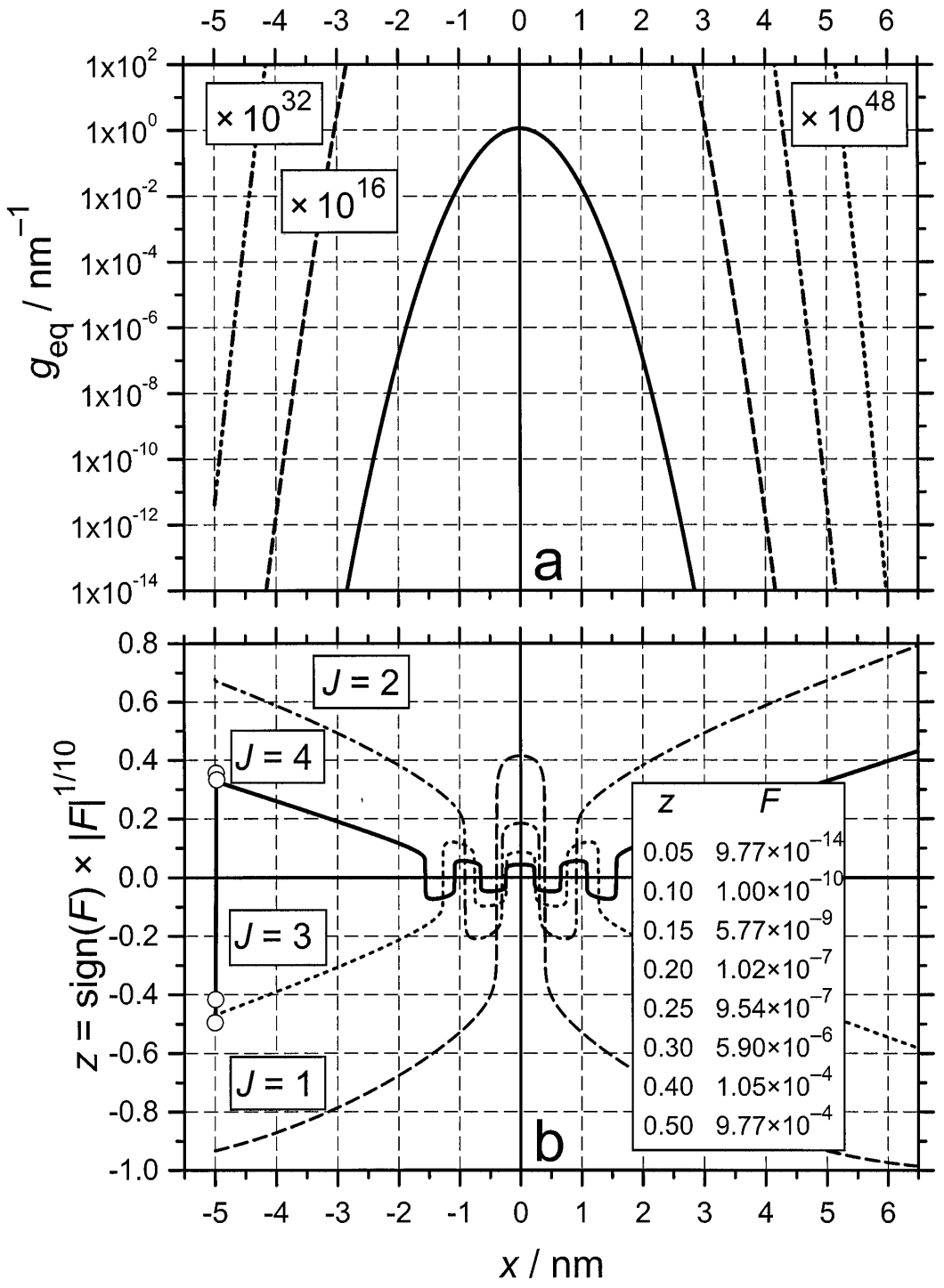

Fig. 1. Example 1: One-dimensional diffusion in a reduced harmonic potential $u(x)=$ $\alpha x^{2}$ with $\alpha=4 \mathrm{~nm}^{2}$ and $D=6 \times 10^{9} \mathrm{~nm}^{2} \mathrm{~s}^{-1}(h=0.01 \mathrm{~nm}$, reflecting boundaries at $X_{0}=-5.005 \mathrm{~nm}$ and $X_{N}=9.005 \mathrm{~nm}$ ). (a) Equilibrium distribution $g_{\mathrm{eq}}(x)$, calculated with Eq. (47). (b) Relative deviations $F$ (see Eq. (48)) of numerical stationary distributions $w_{\mathrm{stat}}(x)$ from the equilibrium distribution $g_{\mathrm{eq}}(x)$ in the $z$-representation (see Eq. (49)); $w_{\text {stat }}(x)$ depends on the degree $2 J$ of the interpolation polynomials; for $J=4$, the four leftmost points $z_{i}(i=1,2,3,4)$ are represented by open circles. $w_{\text {stat }}(x)$ is independent of the start position $x_{n}$ and the length of the first time step $t_{\mathrm{a}}$ (as long as $t_{\mathrm{a}}$ is below the stability limit) with an accuracy of 13 digits. $w_{\text {stat }}(x)$ is reached at the time $t_{\text {stat }}=t_{\mathrm{a}} \times 2^{18}$ $\approx 1.12 \mathrm{~ns}\left(t_{\mathrm{a}} \approx 4.27 \mathrm{fs}\right)$ 


$$
z=\operatorname{sign}(F) \times|F|^{s},
$$

with $s=0.1$ in the present paper. A table with selected values of $z$ and $F(z)$ is shown as inset in Fig. 1b. For simplicity, the quantity $z$ will be also called relative deviation.

In Fig. $1 \mathrm{~b}$ the quantity $z(x)$ is shown for $J=1,2,3,4$. At $x=0 \mathrm{~nm}$, an increase of $J$ by 1 reduces $F$ roughly by a factor of $10^{-3}$ and increases the number of intersections of $z$ with the line $z=0$ by $2 . w_{\text {stat }}(x)$ is independent of the length of the first time step $t_{\mathrm{a}}$. The present data were obtained with $t_{\mathrm{a}} \approx 4.27 \mathrm{fs}$, which corresponds for $w(0,0 \mid 0)$ to a decrease from $100 \mathrm{~nm}^{-1}$ to $\approx 48.8 \mathrm{~nm}^{-1}$. At the time $t_{\text {stat }}=t_{\mathrm{a}} \times 2^{18} \approx 1.12 \mathrm{~ns}$ the numerical curves $w\left(x, t_{\text {stat }} \mid x_{n}\right)$ were independent of $x_{n}$ with an accuracy of 13 digits.

Independently of the neglect of extremely small terms, the accuracy of the computation of $w\left(x, t \mid x_{n}\right)$ is limited by the accumulation of round-off errors, which entails a deviation of the normalization sums, $S_{\text {norm, }}$, from unity. In the present case, at $t_{\text {stat }} \approx 1.12 \mathrm{~ns}$, the deviation of $S_{\text {norm }}$, from unity, $S_{\text {norm }}-1 \approx 3 \times 10^{-12}$, is about 150 times larger than the true value of $F(0) \approx 2 \times 10^{-14}$ for $J=4$. This error is corrected for by a renormalization of the numerical fundamental solutions after every 10 time-doubling cycles.

With $J=4$, the relative deviation $|F|$ is less than $10^{-4}$ in a range of $g_{\text {eq }}$ of about 62 orders of magnitude $(z(6 \mathrm{~nm})=0.4$ corresponds to $F(6 \mathrm{~nm}) \approx$ $1 \times 10^{-4}$ in Fig. $1 \mathrm{~b}$ and $g_{\text {eq }}(6 \mathrm{~nm}) / g_{\text {eq }}(0 \mathrm{~nm}) \approx 10^{-62}$ in Fig. $\left.1 \mathrm{a}\right)$. The only strongly irregular data points are the four leftmost points $z_{i}(i=1,2,3,4)$, which are represented by open circles in Fig. 1b. They are a consequence of the fact that $g_{\text {eq }}$ does not conform to the boundary condition Eq. (34). Finally, this computation illustrates the numerical stability of the algorithm for a value of $t_{\mathrm{a}}$ close to the upper stability limit.

The present algorithm is only of first order with respect to time. Hence one may expect a strong increase of accuracy by reducing the length of the first time step $t_{\mathrm{a}}$. Let $\left(t_{\mathrm{a}}\right)_{m}$ be defined by $\left(t_{\mathrm{a}}\right)_{m}=\left(t_{\mathrm{a}}\right)_{0} \times 2^{-m}$, where $\left(t_{\mathrm{a}}\right)_{0}$ is the time step used in the calculation of $w_{\text {stat }}(x)$ in Fig. 1 and $m=0,1$, $2, \ldots$ In Fig. 2 the results are shown for $J=4$ and a rather short time $t \approx 1.07 \mathrm{ps}$, when the center of $g\left(x, t \mid x_{n}\right)(\approx 7.6 \mathrm{~nm})$ is still close to the start position $x_{n}=8.0 \mathrm{~nm}$. The pattern of the relative deviations $z\left(x_{i}\right)$ initially strongly changes with increasing $m$ and finally becomes virtually constant for $m \geq 24$. $|F|$ is less than $10^{-4}$ in a range of about 8 orders of magnitude of $g\left(x, t \mid x_{n}\right)$. Close to the center of $g\left(x, t \mid x_{n}\right),|F|$ is $<10^{-8}$.

The analogous results for $t \approx 8.53 \mathrm{ps}$ are shown in Fig. 3. Relative to the results in Fig. 2, with $m=36$ a much shorter first time step is needed for the attainment of a constant pattern of $z$, and at the same time $|F|$ is less than $10^{-4}$ in a range of about 34 orders of magnitude of $g\left(x, t \mid x_{n}\right)$. Close to the center of $g\left(x, t \mid x_{n}\right),|F|$ is $<10^{-11}$. Finally it should be noted that $g\left(x, t \mid x_{n}\right)$ in Fig. 3 approximately corresponds to the rightmost curve in Fig. 1 of ref. [19], where the same example is used for the illustration of the gain of accuracy by the so-called virtual gridding technique. 


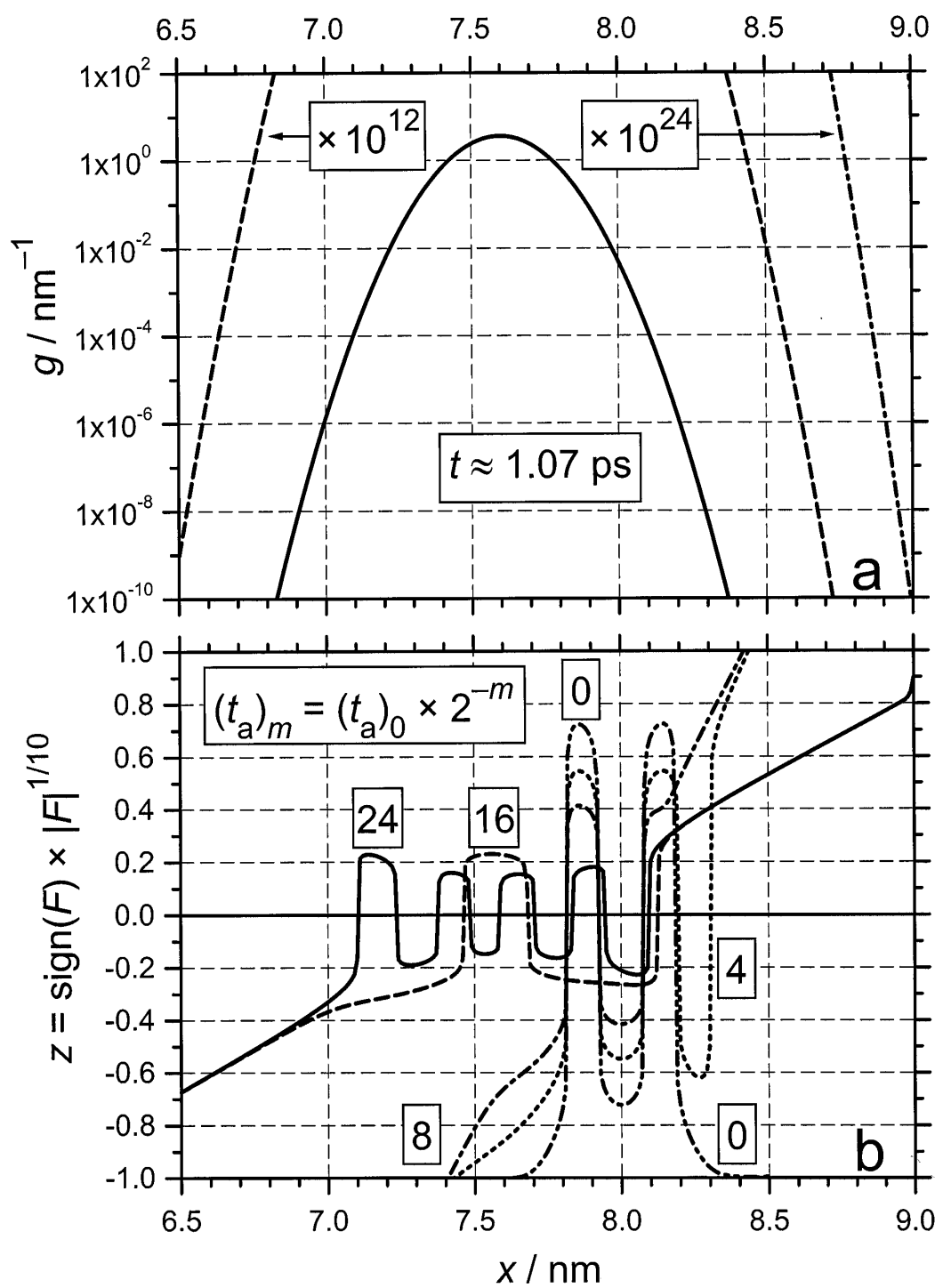

Fig. 2. Example 1: One-dimensional diffusion in a reduced harmonic potential $u(x)=$ $\alpha x^{2}$ with $\alpha=4 \mathrm{~nm}^{2}$ and $D=6 \times 10^{9} \mathrm{~nm}^{2} \mathrm{~s}^{-1}(h=0.01 \mathrm{~nm}$, reflecting boundaries at $X_{0}=-5.005 \mathrm{~nm}$ and $X_{N}=9.005 \mathrm{~nm}$ ). (a) Fundamental solution $g\left(x_{i}, t \mid x_{n}\right)$, calculated with Eq. (47) with $x_{n}=8 \mathrm{~nm}$ and $t \approx 1.07 \mathrm{ps}$. (b) Relative deviations of the numerical fundamental solution $w\left(x_{i}, t \mid x_{n}\right)$ from $g\left(x_{i}, t \mid x_{n}\right)$. w( $\left.x_{i}, t \mid x_{n}\right)$ was calculated with $J=4$ and different values of $t_{\mathrm{a}}=\left(t_{\mathrm{a}}\right)_{0} \times 2^{-m}$, where $\left(t_{\mathrm{a}}\right)_{0} \approx 4.27 \mathrm{fs}$. The numbers in frames are the values of $m$. 


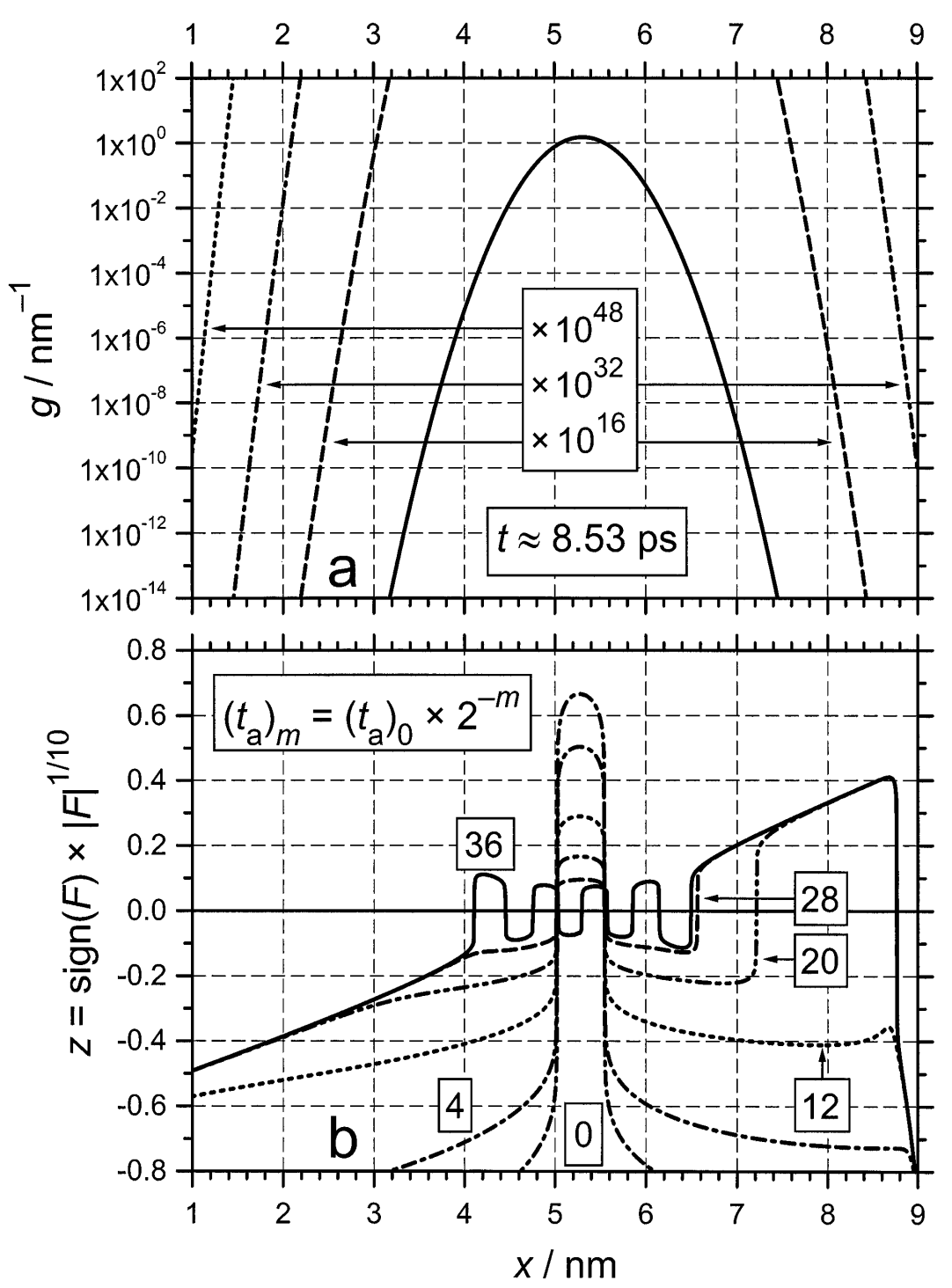

Fig. 3. Example 1: One-dimensional diffusion in a reduced harmonic potential $u(x)=$ $\alpha x^{2}$ with $\alpha=4 \mathrm{~nm}^{2}$ and $D=6 \times 10^{9} \mathrm{~nm}^{2} \mathrm{~s}^{-1}(h=0.01 \mathrm{~nm}$, reflecting boundaries at $X_{0}=-5.005 \mathrm{~nm}$ and $X_{N}=9.005 \mathrm{~nm}$ ). (a) Fundamental solution $g\left(x_{i}, t \mid x_{n}\right)$, calculated with Eq. (47) with $x_{n}=8 \mathrm{~nm}$ and $t \approx 8.53$ ps. (b) Relative deviations $z$ of the numerical fundamental solution $w\left(x_{i}, t \mid x_{n}\right)$ from $g\left(x_{i}, t \mid x_{n}\right) . w\left(x_{i}, t \mid x_{n}\right)$ was calculated with $J=4$ and different values of $t_{\mathrm{a}}=\left(t_{\mathrm{a}}\right)_{0} \times 2^{-m}$, where $\left(t_{\mathrm{a}}\right)_{0} \approx 4.27 \mathrm{fs}$. The numbers in frames are the values of $m$. 


\subsection{Negative effect of detailed balance on the accuracy of the algorithm}

As already mentioned in the Introduction, the exact detailed balance is considered by some authors $[15,16,19]$ to be a necessary condition for a good diffusion algorithm. In the case of a harmonic potential it can be easily demonstrated that, on the contrary, the enforced detailed balance leads to completely wrong numerical solutions, if the potential range of interest is very large like in example 1 . Two sets of numerical curves $w$ were computed with $J=1$. The first set corresponds to the present algorithm. The second set satisfies the additional requirement of detailed balance, which is easily implemented by a redefinition of the quantities $H_{n, j}$. By assigning the index $n=0$ to $x=0$, the new set of $H_{n, j}$ is obtained by the identity

$$
\left(H_{0, j}\right)_{\text {new }}=\left(H_{0, j}\right)_{\text {old }} \quad(j=0, \pm 1) \text {, }
$$

and the recursion formulae:

$$
\begin{aligned}
& \left(H_{n+1,-1}\right)_{\text {new }} \exp \left(-\alpha x_{n+1}^{2}\right)=\left(H_{n,+1}\right)_{\text {new }} \exp \left(-\alpha x_{n}^{2}\right) \quad(n \geq 0), \\
& \left(H_{n+1,-1}\right)_{\text {new }}=\left(H_{n,+1}\right)_{\text {new }} \exp \left(+\alpha h^{2}(2 n+1)\right), \\
& \eta=\left(H_{n+1,-1}\right)_{\text {new }} /\left(H_{n+1,-1}\right)_{\text {old }}, \\
& \left(H_{n+1, j}\right)_{\text {new }}=\left(H_{n+1, j}\right)_{\text {old }} \times \eta \quad(j=0, \pm 1) .
\end{aligned}
$$

The calculation of $\left(H_{n, j}\right)_{\text {new }}$ with $n \leq-1$ is analogous.

In Fig. 4 the results are shown. Quantities referring to detailed balance are labeled with an asterisk. In the logarithmic plot of Fig. 4 a, the curves $w$ and $g$ virtually coincide in the upper range of two orders of magnitude and cannot be distinguished from each other for arbitrary $\left|x_{n}\right| \leq 8 \mathrm{~nm}$ and $t \leq$ 1.07 ps. The curves $w^{*}$ differ already significantly from the curves $g$ for $x_{n}=-4 \mathrm{~nm}$ and are completely wrong for $x_{n}=8 \mathrm{~nm}$. The latter wrong result is not surprising because of the accumulation of a very large systematic error in the quantities $\left(H_{n, j}\right)_{\text {new. }}$. In Fig. 4 a the ratio $\eta$ is plotted (see Eq. $(51 \mathrm{c})): \eta \approx 0.75$ for $x_{n}=-4 \mathrm{~nm}$ and $\eta \approx 10^{-2}$ for $x_{n}=+8 \mathrm{~nm}$. In Fig. $4 \mathrm{~b}$ and $4 \mathrm{c}$ the relative deviations $z$ of $w$ from $g$ are shown for $x_{n}=$ $8 \mathrm{~nm}$ and $x_{n}=-4 \mathrm{~nm}$. The smallest deviations are always found close to the maximum of each curve $g$ (the three open circles correspond to the indices $i_{\max }, i_{\max } \pm 10$ ).

With $x_{n}=0 \mathrm{~nm}$, the initial differences between $w$ and $w^{*}$ are very small. Significant differences between $z$ and $z^{*}$ occur only at times, when $w$ is already very close to the stationary distribution $w_{\text {stat. }}$ The final stationary distribution $w_{\text {stat }}^{*}$ is reached at the time $t_{13} \approx 8.74 \mathrm{~ns}$ and agrees with $g_{\text {eq }}$ with a relative error $|F|<10^{-12}$ in the whole range of $x_{i}$ except for the three rightmost points $(i=N-2, N-1, N)$, where $|F|<10^{-10}$ (note that the range of $g$ covers 140 orders of magnitude!). For comparison, the final stationary distribution $w_{\text {stat }}$ is reached already after $1.09 \mathrm{~ns}$ ( $w_{\text {stat }}$ is independent of $x_{n}$ with an accuracy of 13 digits). 


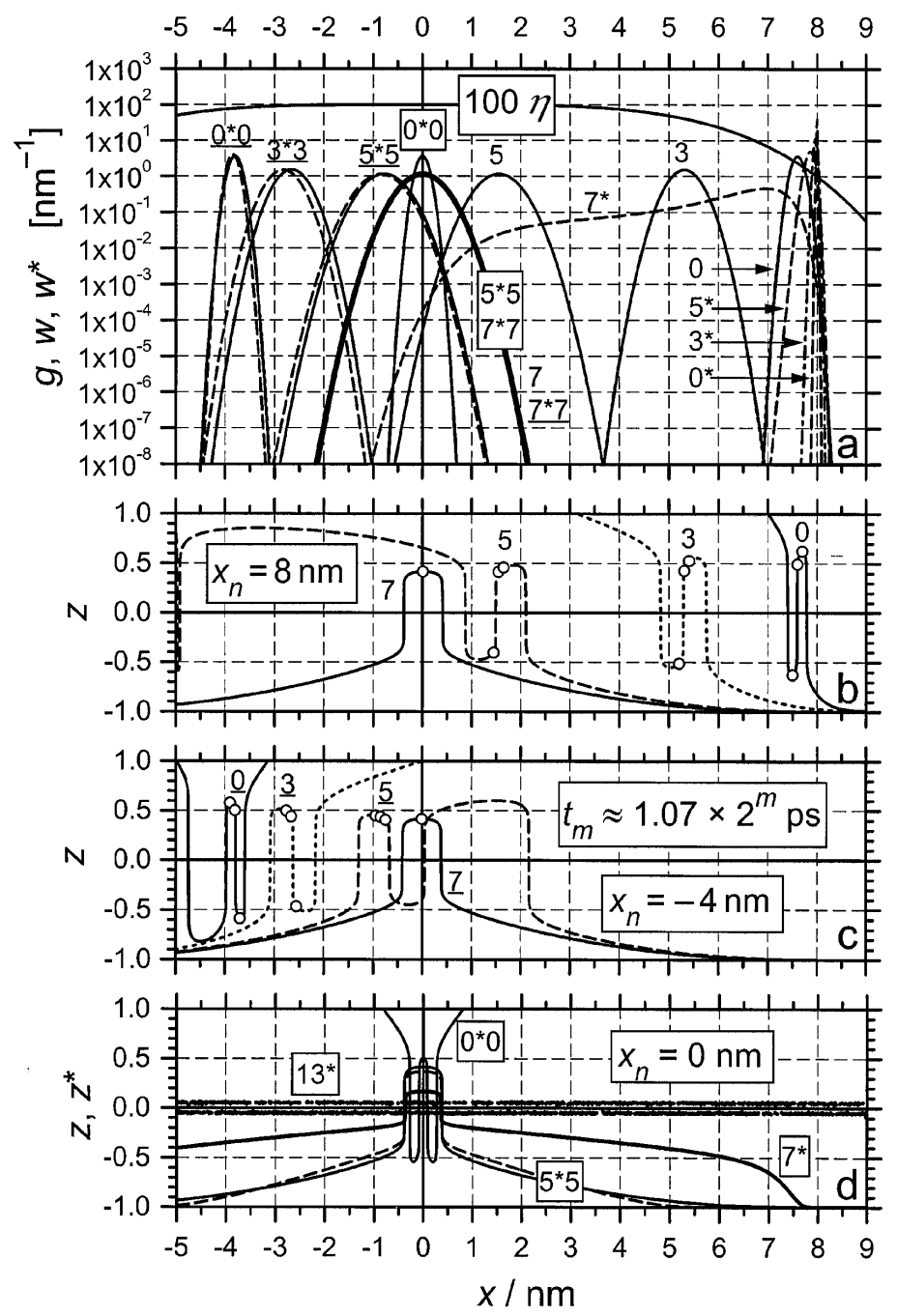

Fig. 4. Example 1: One-dimensional diffusion in a reduced harmonic potential $u(x)=$ $\alpha x^{2}$ with $\alpha=4 \mathrm{~nm}^{2}$ and $D=6 \times 10^{9} \mathrm{~nm}^{2} \mathrm{~s}^{-1}$; negative effect of a detailed balance on the accuracy of the numerical fundamental solutions calculated with $J=1$. (a) Three sets of curves are shown, referring to the start positions $x_{n}=8 \mathrm{~nm}, x_{n}=-4 \mathrm{~nm}$ (underlined numbers), and $x_{n}=0 \mathrm{~nm}$ (numbers in frames). The numbers $m=0,3,5,7,13$ refer to the times $t_{m} \approx 1.07 \times 2^{m}$ ps. The curves $g(-)$ and $w(\cdots)$ virtually coincide and cannot be distinguished from each other; the curves corresponding to $t_{7}$ virtually coincide with the stationary distribution (thick solid curve). The curves $w^{*}(---)$ correspond to a detailed balance. $\eta$ is defined by Eq. (51c). (b) Relative deviation $z$ of $w$ from $g$ for $x_{n}=8 \mathrm{~nm}$. (c) Relative deviation $z$ of $w$ from $g$ for $x_{n}=-4 \mathrm{~nm}$. (d) Relative deviations $z(---)$ and $z^{*}(-)$ of $w$ and $w^{*}$ from $g$ for $x_{n}=0 \mathrm{~nm}$. The stationary distribution $w_{\text {stat }}^{*}$ was reached at $t_{13} \approx 8.74 \mathrm{~ns}$; the corresponding data points $z_{\text {stat }}^{*}$ are statistically distributed relative to $z=0$ with $\left|z_{\text {stat }}^{*}\right| \approx 0.05$. 


\subsection{Example 2: Diffusion in a double-minimum potential with reflecting boundaries}

A double minimum potential with reflecting boundaries at $x=X_{0}$ and $x=$ $X_{N}=-X_{0}$ is defined by a symmetrical polynomial of the $8^{\text {th }}$ degree:

$$
u(x)=u(0)\left[1-8\left(x / X_{N}\right)^{2}+20\left(x / X_{N}\right)^{4}-16\left(x / X_{N}\right)^{6}+4\left(x / X_{N}\right)^{8}\right] .
$$

(This polynomial is easily obtained by a transformation of the Chebyshev polynomial $T_{8}(x)[21,23]$.) The following computations were performed with $X_{0}=-8.005 \mathrm{~nm}, X_{N}=8.005 \mathrm{~nm}, N=1601, h=0.01 \mathrm{~nm}$, and $u(0)$ $\approx 34.539$, which corresponds to an equilibrium distribution extending over 15 orders of magnitude. The results in Fig. 5 illustrate four aspects of the algorithm:

(a) The probability conservation remains exact if the potential is given by a polynomial of a degree $\leq 2 J+1$.

(b) The systematic errors of the stationary numerical solution close to a reflecting boundary are very small, if the potential at the boundary satisfies Eq. (35).

(c) If the barrier between the two potential minima is high, two very different time scales can be distinguished (as is well known from Kramers's work [31, 32]). If $x_{1} \leq x_{n}<0 \mathrm{~nm}$, then in the left part of the potential a quasi-stationary distribution is attained in about $1 \mathrm{~ns}$. The attainment of the final equilibrium distribution is extremely slow: Close to the left potential minimum, the difference $w\left(x_{i}, t \mid x_{n}\right)-w_{\text {stat }}\left(x_{i}\right)$ decays exponentially almost exactly, with a time constant $\tau_{\text {stat }} \approx 7.28 \times 10^{4} \mathrm{~s}$. At the time $t_{\text {stat }} \approx$ $2.46 \times 10^{6} \mathrm{~s} \approx 33.8 \tau_{\text {stat }}$, all $N$ fundamental solutions $w\left(x_{i}, t \mid x_{n}\right)$ were equal with an accuracy of 13 digits. The last time step of $1.23 \times 10^{6} \mathrm{~s}$ was by a factor of $2^{108} \approx 3.245 \times 10^{32}$ longer than the first time step, $t_{\mathrm{a}} \approx 3.79 \times$ $10^{-27} \mathrm{~s}$. Note that this problem cannot be solved with a standard method, since even in the most favorable case, where $t_{\mathrm{a}} \approx 4.27 \times 10^{-15} \mathrm{~s}$ is already close to the stability limit of the algorithm and $t_{\text {stat }} \approx 5 \tau_{\text {stat }} \approx 3.64 \times 10^{5} \mathrm{~s}$, about $10^{20}$ time steps would be needed.

(d) The agreement of the stationary numerical distribution, $w_{\text {stat }}(x)$, with the exact equilibrium distribution, $g_{\text {eq }}(x)$, is very good. $|F|$ is less than $10^{-11}$ in the vicinity of the maxima of $g_{\mathrm{eq}}(x)$, less than $10^{-10}$ in a range of more than 5 orders of magnitude of $g_{\text {eq }}(x)$, and less than $10^{-8}$ everywhere except for $x_{1}$ and $x_{N}$, where $F \approx 3 \times 10^{-8}$. The lack of complete mirror symmetry of the curve $z(x)$ in Fig. $5 \mathrm{~b}$ is caused by the insufficient numerical accuracy.

\section{Spherically symmetric three-dimensional diffusion}

The treatment of spherically symmetric three-dimensional diffusion is essentially analogous to that of one-dimensional diffusion. Therefore, in the following only the peculiar aspects of three-dimensional diffusion are treated in detail. 


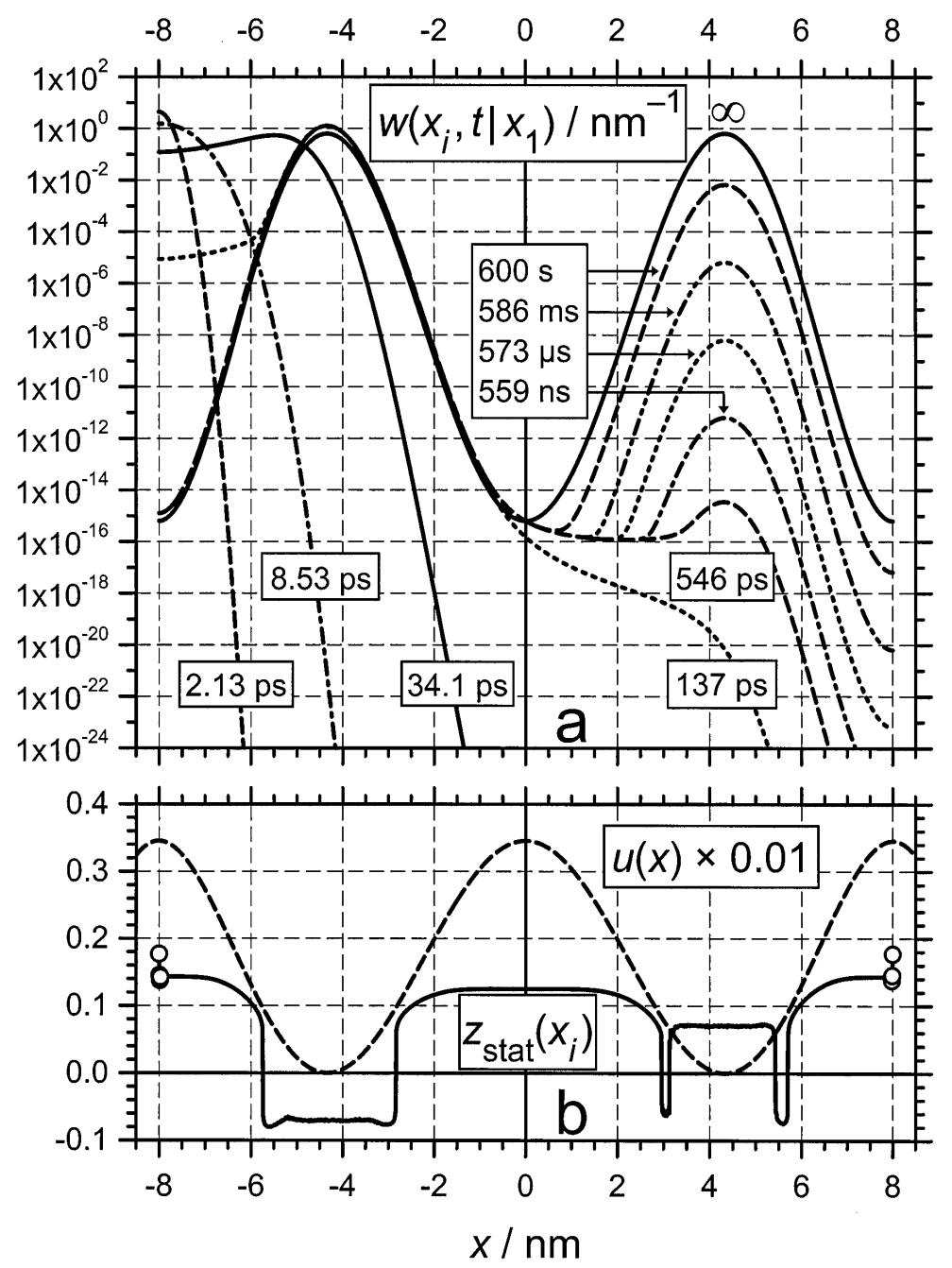

Fig. 5. Example 2: One-dimensional diffusion in a reduced double minimum potential $u(x)$ defined by Eq. (52): $X_{0}=-8.005 \mathrm{~nm}, X_{N}=8.005 \mathrm{~nm}, N=1601, h=0.01 \mathrm{~nm}$, $u(0) \approx 34.539, D=6 \times 10^{9} \mathrm{~nm}^{2} \mathrm{~s}^{-1}$. (a) Numerical fundamental solutions $w$ (first time step $t_{\mathrm{a}} \approx 3.79 \times 10^{-27} \mathrm{~s}$ ). (b) Reduced potential $u(x)$ and relative deviation $z_{\text {stat }}$ of the stationary distribution $w_{\text {stat }}$ from the equilibrium distribution $g_{\text {eq }}$.

\subsection{Definition of spherical shells and mean radii}

Let a sphere of radius $R_{0}$ be surrounded by a series of concentric spherical surfaces with increasing radii $R_{1}, R_{2}, \ldots, R_{i}, \ldots, R_{N-1}, R_{N}$ with

$$
R_{i}=R_{i-1} \times f_{i}
$$


The volume of the $i$-th spherical shell between the radii $R_{i-1}$ and $R_{i}$ is

$$
V_{i}=(4 \pi / 3)\left(R_{i}^{3}-R_{i-1}^{3}\right) .
$$

As effective radius $r_{i}$ of the $i$-th spherical shell the mean radius of the shell is chosen:

$$
r_{i}=\langle r\rangle_{V_{i}}=\frac{4 \pi}{3 V_{i}} \int_{R_{i-1}}^{R_{i}} r^{3} \mathrm{~d} r
$$

In the important special case of constant $f$, Eqs. (53) to (55) can be written as follows:

$$
\begin{aligned}
& R_{i}=R_{0} \times f^{i}, \\
& v_{i}=(4 \pi / 3) R_{0}^{3} f^{3 i-3}\left(f^{3}-1\right)=V_{i} f^{3 i-3}, \\
& r_{i}=R_{0}\left(\frac{3\left(f^{4}-1\right)}{4\left(f^{3}-1\right)}\right) f^{i-1}=\langle r\rangle_{V_{1}} f^{i-1} .
\end{aligned}
$$

If the initial radial increments must be very small and the maximum radius $r_{N}$ is very large, different values of $f$ can be used for the near zone and for the far zone: $f_{1}=1+q_{1}$ and $f_{2}=1+q_{2}$ with $q_{1}<q_{2}$. Let $f=f(i)$ be a continuous function of the index $i$. A necessary condition for a smooth transition from $f_{1}$ to $f_{2}$ is that the second derivative of $f(i)$ with respect to $i$ be continuous. The following function $f(i)$ has this property and turns out to be flexible enough for the present purpose:

$$
f(i)=f_{1}+\left(f_{2}-f_{1}\right)\left(1-\exp \left(-\left(i / i_{1-2}\right)\left[1-\exp \left(-i / i_{1-2}\right)\right]^{\kappa}\right)\right) .
$$

Here the index $i_{1-2}$ roughly defines the beginning transition from $f_{1}$ to $f_{2}$ and the exponent $\kappa$ defines the steepness of that transition. An application of Eq. (56) is presented in section 3.6 (the corresponding function $f(i)$ is shown in Fig. 9b). Other functions $f(i)$ were also tested. A single polynomial and a combination of two polynomials (for different ranges of $i$ ) turned out to be unsuitable.

\subsection{Fundamental solutions and probability conservation}

Let a fundamental solution at the time $t=t_{\mathrm{a}}, w\left(r_{i}, t_{\mathrm{a}} \mid r_{n}\right)$, be defined by Eq. (57):

$$
w\left(r_{i}, t_{\mathrm{a}} \mid r_{n}\right) \approx w\left(r_{i}, 0 \mid r_{n}\right)+\left(\frac{\partial w\left(r_{i}, t \mid r_{n}\right)}{\partial t}\right)_{t=0} \times t_{\mathrm{a}} .
$$

$w\left(r_{i}, O \mid r_{n}\right)$ is defined by Eq. (2) and $\left[\partial w\left(r_{i}, t \mid r_{n}\right) / \partial t\right]_{t=0}$ is calculated with Eq. (1). The required first and second derivatives with respect to $r, w^{\prime}\left(r_{i}, 0 \mid\right.$ $\left.r_{n}\right)$ and $w^{\prime \prime}\left(r_{i}, 0 \mid r_{n}\right)$, are again defined by a set of $2 J+1$ polynomials $y_{n+j, n}(r)$ of the degree $2 J$ with $j=0, \pm 1, \ldots, \pm J$ : 


$$
\begin{aligned}
& w^{\prime}\left(r_{n+j}, 0 \mid r_{n}\right)=V_{n}^{-1} y_{n+j, n}^{\prime}\left(r_{n+j}\right), \\
& w^{\prime \prime}\left(r_{n+j}, 0 \mid r_{n}\right)=V_{n}^{-1} y_{n+j, n}^{\prime \prime}\left(r_{n+j}\right) .
\end{aligned}
$$

With $J=2$, the $2 J+1=5$ polynomials are

$$
\begin{aligned}
& y_{n, n}(r)=A_{n, 0}\left(r-r_{n-2}\right)\left(r-r_{n-1}\right)\left(r-r_{n+1}\right)\left(r-r_{n+2}\right), \\
& y_{n+1, n}(r)=A_{n,+1}\left(r-r_{n-1}\right)\left(r-r_{n+1}\right)\left(r-r_{n+2}\right)\left(r-r_{n+3}\right), \\
& y_{n-1, n}(r)=A_{n,-1}\left(r-r_{n-3}\right)\left(r-r_{n-2}\right)\left(r-r_{n-1}\right)\left(r-r_{n+1}\right), \\
& y_{n+2, n}(r)=A_{n,+2}\left(r-r_{n+1}\right)\left(r-r_{n+2}\right)\left(r-r_{n+3}\right)\left(r-r_{n+4}\right), \\
& y_{n-2, n}(r)=A_{n,-2}\left(r-r_{n-4}\right)\left(r-r_{n-3}\right)\left(r-r_{n-2}\right)\left(r-r_{n-1}\right) .
\end{aligned}
$$

The factors $A_{n, j}$ are obtained from the condition that all five polynomials pass through the point $y_{n, n}\left(r_{n}\right)$ :

$$
y_{n+j, n}\left(r_{n}\right)=y_{n, n}\left(r_{n}\right)=1 \quad(j=0, \pm 1, \pm 2) .
$$

For the calculation of the fundamental solutions $w\left(r_{n+j}, t_{\mathrm{a}} \mid r_{n}\right)$ with $j=0$, $\pm 1, \ldots, \pm J$, the quantities

$$
H_{n, j} \equiv V_{n}\left[\partial w\left(r, t \mid r_{n}\right) / \partial t\right]_{r=r_{n+j}, t=0}
$$

are needed, which are according to Eq. (1) and with $u \equiv U / k_{\mathrm{B}} T$ and $k=0$ :

$$
\begin{aligned}
H_{n, j}= & D\left(r_{n+j}\right) y_{n+j, n}^{\prime \prime}\left(r_{n+j}\right)+\left[\left(2 / r_{n+j}\right) D\left(r_{n+j}\right)+D^{\prime}\left(r_{n+j}\right)\right. \\
& \left.+D\left(r_{n+j}\right) u^{\prime}\left(r_{n+j}\right)\right] y_{n+j, n}^{\prime}\left(r_{n+j}\right)+\left[\left(2 / r_{n+j}\right) D\left(r_{n+j}\right) u^{\prime}\left(r_{n+j}\right)\right. \\
& \left.+D^{\prime}\left(r_{n+j}\right) u^{\prime}\left(r_{n+j}\right)+D\left(r_{n+j}\right) u^{\prime \prime}\left(r_{n+j}\right)\right] y_{n+j, n}\left(r_{n+j}\right) .
\end{aligned}
$$

In the special case of a constant potential and a constant relative diffusion coefficient, Eq. (67) reduces to

$$
H_{n, j}=D\left[y_{n+j, n}^{\prime \prime}\left(r_{n+j}\right)+\left(2 / r_{n+j}\right) y_{n+j, n}^{\prime}\left(r_{n+j}\right)\right] .
$$

The probability balance is now defined by

$$
S_{n}=\sum_{j=-J}^{+J} H_{n, j} V_{n+j} .
$$

In contrast to one-dimensional diffusion with constant intervals $\Delta x$, the probability balance $S_{n}$ is no longer exactly equal to zero. The relative deviation of $S_{n}$ from zero is defined by

$$
\omega_{n}=S_{n} /\left|H_{n, 0} V_{n}\right| .
$$

In the special case of constant $u$ and $D$ (cf. Eq. (68)) and a constant radial increment factor $f \equiv 1+q, \omega$ is independent of $n$. By using the definitions of $V_{i}$ and $r_{i}$ in Eqs. (54a) and (55a), the following expressions for $\omega_{n}$ can be derived for $J=1,2$ : 
Table 2. Relative deviation $\left(\omega_{n}\right)_{2 J}(J=1,2,3,4)$ of the three-dimensional diffusion balance from zero for different values of $q$. $\left(\omega_{n}\right)_{2}$ and $\left(\omega_{n}\right)_{4}$ were calculated with Eqs. (71) and (72), respectively. The values of $\left(\omega_{n}\right)_{6}$ and $\left(\omega_{n}\right)_{8}$ are the results of purely numerical calculations.

\begin{tabular}{lllll}
\hline$q$ & $\left(\omega_{n}\right)_{2}$ & $\left(\omega_{n}\right)_{4}$ & $\left(\omega_{n}\right)_{6}$ & $\left(\omega_{n}\right)_{8}$ \\
\hline 0.001 & $-9.98 \times 10^{-13}$ & $+6.38 \times 10^{-18}$ & & \\
0.002 & $-1.59 \times 10^{-11}$ & $+4.07 \times 10^{-16}$ & & \\
0.003 & $-8.05 \times 10^{-11}$ & $+4.62 \times 10^{-15}$ & & \\
0.004 & $-2.54 \times 10^{-10}$ & $+2.59 \times 10^{-14}$ & & \\
0.006 & $-1.28 \times 10^{-9}$ & $+2.93 \times 10^{-13}$ & & \\
0.008 & $-4.03 \times 10^{-9}$ & $+1.64 \times 10^{-12}$ & & \\
0.010 & $-9.80 \times 10^{-9}$ & $+6.21 \times 10^{-12}$ & & \\
0.015 & $-4.92 \times 10^{-8}$ & $+6.98 \times 10^{-11}$ & & +8. \\
0.020 & $-1.54 \times 10^{-7}$ & $+3.86 \times 10^{-10}$ & $-1.9 \times 10^{-13}$ \\
0.025 & $-3.72 \times 10^{-7}$ & $+1.45 \times 10^{-9}$ & $-1.10 \times 10^{-11}$ & \\
0.030 & $-7.64 \times 10^{-7}$ & $+4.28 \times 10^{-9}$ & $-4.64 \times 10^{-11}$ & \\
0.040 & $-2.37 \times 10^{-6}$ & $+2.34 \times 10^{-8}$ & $-4.48 \times 10^{-10}$ & $+1.41 \times 10^{-11}$ \\
0.060 & $-1.16 \times 10^{-5}$ & $+2.53 \times 10^{-7}$ & $-1.08 \times 10^{-8}$ & $+7.55 \times 10^{-10}$ \\
0.080 & $-3.53 \times 10^{-5}$ & $+1.35 \times 10^{-6}$ & $-1.01 \times 10^{-7}$ & $+1.25 \times 10^{-8}$ \\
0.100 & $-8.34 \times 10^{-5}$ & $+4.93 \times 10^{-6}$ & $-5.70 \times 10^{-7}$ & $+1.10 \times 10^{-7}$ \\
0.150 & $-3.90 \times 10^{-4}$ & $+5.06 \times 10^{-5}$ & $-1.30 \times 10^{-5}$ & $+5.63 \times 10^{-6}$ \\
0.200 & $-1.15 \times 10^{-3}$ & $+2.60 \times 10^{-4}$ & $-1.19 \times 10^{-4}$ & $+9.40 \times 10^{-5}$ \\
0.250 & $-2.61 \times 10^{-3}$ & $+9.23 \times 10^{-4}$ & $-6.70 \times 10^{-4}$ & $+8.78 \times 10^{-4}$ \\
\hline
\end{tabular}

$$
\begin{aligned}
& \left(\omega_{n}\right)_{2 J=2}=\frac{-q^{4}}{1+2 q-q^{3}} \\
& \left(\omega_{n}\right)_{2 J=4}=\frac{32 q^{6}\left(1+4 q+\frac{22}{3} q^{2}+8 q^{3}+\frac{271}{48} q^{4}+\frac{21}{8} q^{5}+\frac{25}{32} q^{6}+\frac{13}{96} q^{7}+\frac{1}{96} q^{8}\right)}{5(1+q)^{3}\left(1+q+\frac{1}{2} q^{2}\right)\left(1+q+\frac{1}{3} q^{2}\right)\left(1+2 q-\frac{6}{5} q^{2}-\frac{11}{5} q^{3}-\frac{3}{5} q^{4}\right)} .
\end{aligned}
$$

In Table 2 values of $\left(\omega_{n}\right)_{2 J}$ are listed for $J=1,2,3,4$ and different values of $q$. For $J \geq 2,\left(\omega_{n}\right)_{2 J}$ is extremely small in the whole range of $q$ that is of practical interest. The purely numerically calculated values of $\left(\omega_{n}\right)_{6}$ and $\left(\omega_{n}\right)_{8}$ become rather inaccurate and finally meaningless with decreasing $q$ (as a result of insufficient numerical accuracy). Nevertheless one may estimate that $\left(\omega_{n}\right)_{6}$ and $\left(\omega_{n}\right)_{8}$ are approximately proportional to $q^{8}$ and $q^{10}$, respectively, for small values of $q$. Thus, for small values of $q$, $\left(\omega_{n}\right)_{2 J}$ seems to be approximately proportional to $q^{2 J+2}$ for arbitrary $J \geq 1$.

In the general case of $u=u\left(r_{i}\right), D=D\left(r_{i}\right)$, and $f=f(i)$, additional systematic errors are introduced in the probability balance. However, by a suitable choice of $f=f(i)$ and with $J=4,\left|\left(\omega_{n}\right)_{8}\right|$ can be kept very small. For instance, in the example in section 3.6, the relation $\left|\left(\omega_{n}\right)_{8}\right| \lesssim 1.2 \times 10^{-9}$ was always satisfied. 
The very small error in the probability balance is corrected for by introducing correction factors $\varphi$. Since it is unknown how the deviation from the exact probability conservation is distributed over the central term $H_{n, 0} V_{n}$ on the one hand and the $2 J$ other terms $H_{n, j} V_{n+j}$ with $j \neq 0$ on the other hand, the following symmetrical definition of $\varphi$ is preferred:

$$
H_{n, 0} V_{n} \times \varphi_{n, 0}+\left(S_{n}-H_{n, 0} V_{n}\right) \times \varphi_{n, j \neq 0}=0
$$

or

$$
\varphi_{n, 0} d \varphi_{n, j \neq 0}=1-\left[S_{n} /\left(H_{n, 0} V_{n}\right)\right] .
$$

The corrected values of $H$ are: $\left(H_{n, 0}\right)_{\text {corr }}=H_{n, 0} \times \varphi_{n, 0}$ and $\left(H_{n, j \neq 0}\right)_{\text {corr }}=$ $H_{n, j \neq 0} \times \varphi_{n, j \neq 0}$ with $j= \pm 1, \pm 2, \ldots, \pm J$. Three possibilities were tested: (a) $\varphi_{n, 0}=1$ and $\varphi_{n, j \neq 0} \neq 1$; (b) $\varphi_{n, 0} \neq 1$ and $\varphi_{n, j \neq 0}=1$; (c) $\varphi_{n, 0}=$ $1 / \varphi_{n, j \neq 0}$. In practice it turned out that the final results were almost independent of the specific correction procedure (in the examples 3, 4, and 5, always the correction (c) was applied). Asymmetric corrections, in which either the terms $H_{n, j \geq 1}$ or the terms $H_{n, j \leq-1}$ were multiplied by a correction factor, gave nearly the same results. In the following the subscript "corr" is omitted, since always corrected values of $H_{n, j}$ are implied.

\subsection{The first diffusion step and boundary conditions}

For calculating the result of the first diffusion step of duration $t_{\mathrm{a}}$, relative changes $b_{n, j}$ of $w\left(r_{n+j}, 0 \mid r_{n}\right)$ are again defined by Eqs. (30) and (31), but with the quantities $H_{n, j}$ now given by Eq. (67) and corrected according to Eq. (73). The boundary conditions in Eqs. (34) - (36) remain also valid if $x$ and $X_{0}$ are replaced by $r$ and $R_{0}$. The only essential change concerns the numerical implementation of the boundary conditions. Since the constant interval $\Delta x=h$ is replaced with the variable volume $V_{i}$ of the spherical shells, Eqs. (37) and (38) are to be replaced by the equations

$$
\begin{aligned}
& b_{1,-1} \times V_{0}+b_{1,-2} \times V_{-1}=c_{0}\left(b_{0,+1} \times V_{1}+b_{0,+2} \times V_{2}\right), \\
& b_{2,-2} \times V_{0}=c_{-1}\left(b_{-1,+2} \times V_{1}\right) .
\end{aligned}
$$

The definition of a constant-concentration boundary by Eqs. (37a)-(42a) remains unchanged.

\subsection{Example 3: Free diffusion near a reflecting or absorbing spherical surface}

Let the inner boundary be again specified (as in section 2.5) by the parameter $c_{\mathrm{BC}}$ with $c_{\mathrm{BC}}=+1$ for reflection and $c_{\mathrm{BC}}=-1$ for absorption. Then, with constant potential and constant relative diffusion coefficient, the common theoretical fundamental solution can be written in the form $[4,33]$ : 


$$
\begin{aligned}
& g\left(r_{i}, t \mid r_{n}\right)=\frac{1}{8 \pi r_{i} r_{n} \sqrt{\pi D t}}\left[\exp \left(-\frac{\left(r_{i}-r_{n}\right)^{2}}{4 D t}\right)+c_{\mathrm{BC}} \exp \left(-\frac{\left(r_{i}+r_{n}-2 R_{0}\right)^{2}}{4 D t}\right)\right. \\
& \left.\times\left(1-\frac{2\left(1+c_{\mathrm{BC}}\right) \sqrt{D t}}{R_{0}} \int_{0}^{\infty} \exp \left(-x^{2}-2\left[\frac{\sqrt{D t}}{R_{0}}+\frac{\left(r_{i}+r_{n}-2 R_{0}\right)}{2 \sqrt{D t}}\right] x\right) d x\right)\right] .
\end{aligned}
$$

Eq. (77) is obtained from Eq. (24) in ref. [4] by equating $k_{\text {act }}=0$ and inserting $c_{\mathrm{BC}}$ before the second term and $\left(1+c_{\mathrm{BC}}\right) / 2$ before the integral, and finally by transforming the complementary error function into the present integral. (The computational disadvantage of the complementary error function is the simultaneous appearance of an exponential function with a positive exponent, which makes it impossible to calculate $g\left(r_{i}, t \mid r_{n}\right)$ for large values of $r_{i}+r_{n}$ or $D t$. The integral was calculated with a numerical accuracy of 15 digits for arbitrary values of the bracketed term in the integrand.)

For the comparison of theoretical curves with numerically calculated curves it is advantageous to apply to theoretical curves $g$ the same normalization as to numerical curves $w$. This is achieved by introducing a factor $(1+\varepsilon)$, which is defined by the equation

$$
(1+\varepsilon(t)) \times \sum_{i=1}^{N} g\left(r_{i}, t \mid r_{n}\right) V_{i}=4 \pi \int_{R_{0}}^{\infty} g\left(r, t \mid r_{n}\right) r^{2} \mathrm{~d} r .
$$

In the following the symbol $g^{*} \equiv(1+\varepsilon) g$ is used. In the present example, $\varepsilon$ is almost independent of time in the time range of interest (from 4 ps to $4 \mathrm{~ns}): \varepsilon \approx 1.244 \times 10^{-5}$. The notation for the fundamental solutions is simplified. Since the values of $w$ and $g^{*}$ always refer to specified values of $t$ and $r_{n}$, the simplified notation is $g_{i}^{*} \equiv g^{*}\left(r_{i}, t \mid r_{n}\right)$ and $w_{i} \equiv w\left(r_{i}, t \mid r_{n}\right)$.

In Fig. 6a six pairs of theoretical curves $g_{i}^{*}$ are shown, which were calculated with $R_{0}=0.8 \mathrm{~nm}, f=1.005, r_{n} \approx 2.00 \mathrm{~nm}, n=184, r_{N} \approx$ $2319 \mathrm{~nm}, N=1600, D=6 \times 10^{9} \mathrm{~nm}^{2} \mathrm{~s}^{-1}$. The solid curves refer to a reflecting boundary at $R_{0}\left(c_{\mathrm{BC}}=+1\right.$ in Eq. (77)), and the dashed curves refer to an absorbing boundary at $R_{0}\left(c_{\mathrm{BC}}=-1\right.$ in Eq. (77)). The curves cover the time range from $\approx 4$ ps (curves 0 ) to 4 ns (curves 10 ) in steps of a factor of 4 . The four leftmost data points of the dashed curves 0,2 , and 4 are represented by circles.

The numerical fundamental solutions $w\left(r_{i}, t \mid r_{n}\right)$ were computed with $J=4$. In the case of a reflecting boundary at $R_{0}$, a very small value of $t_{\mathrm{a}}$ $\left(\approx 4.136 \times 10^{-34} \mathrm{~s}\right)$ was used, and the fundamental solutions $w\left(r_{i}, t \mid r_{n}\right)$ were renormalized after every 10 time-doubling cycles. The relative deviations $F$ of $w$ from $g^{*}$ are defined in analogy to Eq. (48) and again given in the $z$ representation (see Eq. (49)) in Fig. 6b. Curve 0 shows the typical limiting pattern of $z$ for $J=4$ with nine intersections with the $z=0$ line; a further reduction of $t_{\mathrm{a}}$ would leave $z(r)$ virtually unchanged. The only irregular points are the four leftmost points of each curve, due to the imperfect nu- 


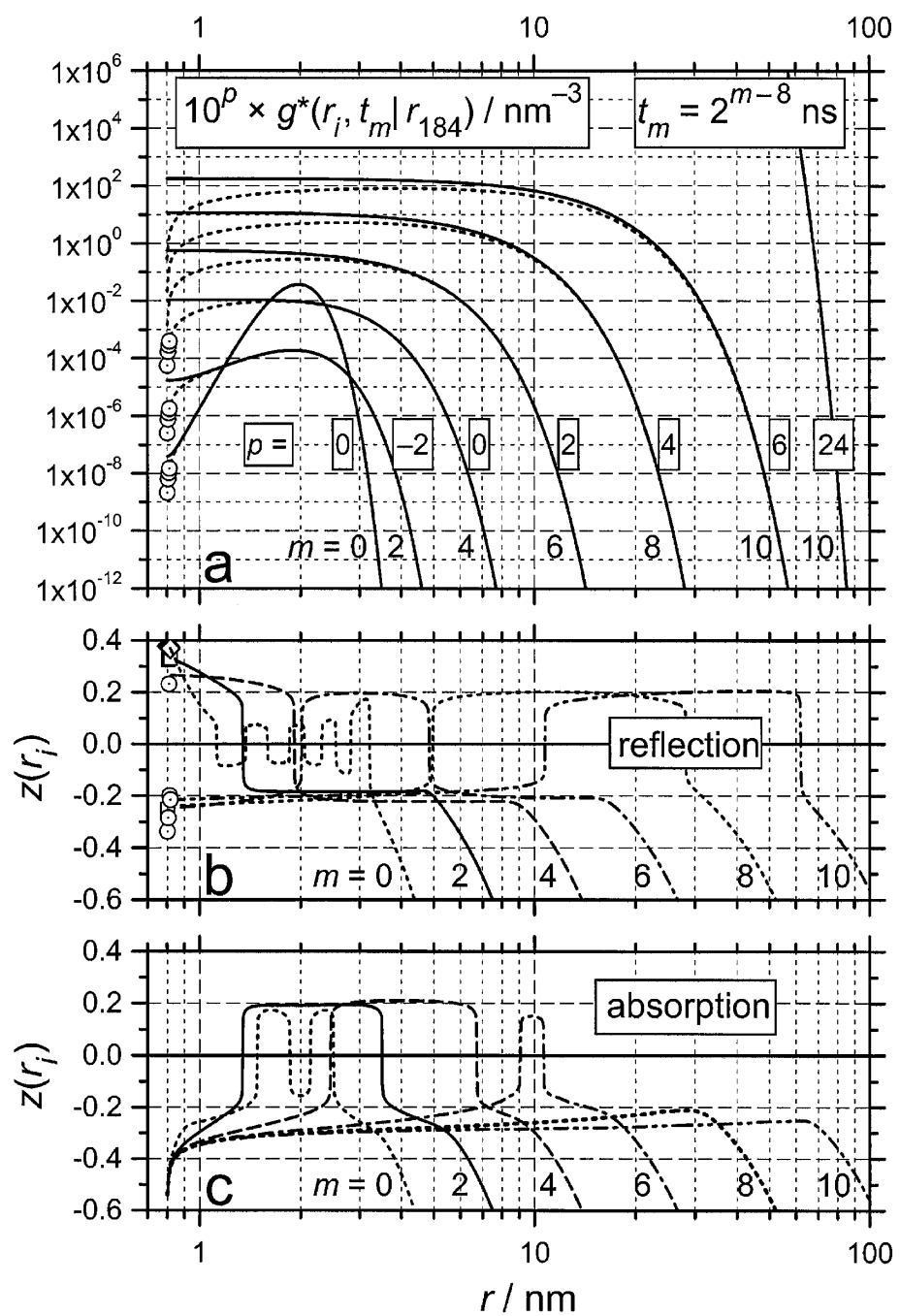

Fig. 6. Example 3: Spherically symmetric three-dimensional diffusion with constant potential and constant relative diffusion coefficient. Parameters: $R_{0}=0.8 \mathrm{~nm}, f=1.005, r_{n}$ $\approx 2.00 \mathrm{~nm}, n=184, r_{N} \approx 2319 \mathrm{~nm}, N=1600, D=6 \times 10^{9} \mathrm{~nm}^{2} \mathrm{~s}^{-1}$; reflecting boundary at $R_{N}$. (a) Theoretical fundamental solutions $g_{i}^{*}$ (see Eqs. (77) and (78)) referring to a reflecting boundary ( - ) or absorbing boundary $(---)$ at $R_{0}$; the curves cover the time range from $t_{0} \approx 4$ ps (curves 0 ) to $t_{10}=4 \mathrm{~ns}$ (curves 10) in steps of a factor of 4 . The four leftmost points of the dashed curves 0,2 , and 4 are represented by open circles. For a better distinction of the curves close to the boundary or the coverage of a larger range of $g^{*}$, the curves with index $m=2,6,8,10$ have been multiplied by suitable factors $10^{p}$. (b) Reflecting boundary: Relative deviations $z$ of $w$ from $g^{*}$ (first time step $t_{\mathrm{a}} \approx$ $4.136 \times 10^{-34} \mathrm{~s}$, renormalization of all $w$ after every 10 time-doubling cycles). (c) Absorbing boundary, relative deviations $z$ of $w$ from $g^{*}\left(t_{\mathrm{a}} \approx 1.164 \times 10^{-19} \mathrm{~s}\right.$, no renormalization). 
merical implementation of the boundary condition. $|F|$ is less than $10^{-4}$ in a range of 15.7 orders of magnitude for $m=0$ and in a range of 31.4 orders of magnitude for $m=10$. At the right side of each curve, $z=-0.4$ or $F \approx-10^{-4}$ corresponds to $g_{i+1}^{*} / g_{i}^{*} \approx 0.5$.

In the case of an absorbing boundary at $R_{0}$, a correction for the accumulation of round-off errors by renormalization can no longer be applied. Therefore, a $2^{48} \approx 2.81 \times 10^{14}$ times larger value of $t_{\mathrm{a}}$ was chosen: $t_{\mathrm{a}} \approx$ $1.164 \times 10^{-19} \mathrm{~s}$. The values of $z$ are shown in Fig. $6 \mathrm{c}$. The only strongly irregular value of $z, z_{1} \approx-0.537$, corresponds to $F_{1} \approx-2.0 \times 10^{-3}$ and is practically independent of time. This rather large value of $F_{1}$ is not surprising in view of the large ratio $g_{2}^{*} / g_{1}^{*} \approx 3$. The accumulation of round-off errors becomes apparent in the $z$-curves for $m=6,8,10$.

\subsection{Example 4: $u=u(r), D=D(r)$, small radial range with constant ratio $r_{i+1} / r_{i}$}

For the present test calculations an arbitrary potential function was chosen:

$$
u(r)=u\left(R_{0}\right) \times \exp \left[-\ln (2)\left|\left(r-R_{0}\right) / \Gamma\right|^{n_{\mathrm{pot}}}\right] \times\left[1+f_{\mathrm{pot}} \ln (2)\left|\left(r-R_{0}\right) / \Gamma\right|^{n_{\mathrm{por}}}\right] .
$$

The second factor is a good approximation to a box potential for high powers $n_{\text {pot }}$. The last factor allows one to introduce additionally a potential barrier. $\Gamma$ is the halfwidth of the potential if $f_{\mathrm{pot}}=0$. For the relative diffusion coefficient the simple expression proposed by Northrup and Hynes [11] was used,

$$
D(r)=D(\infty)\left[1-a_{\text {diff }} \exp \left(-\left(r-R_{0}\right) / R_{0}\right)\right],
$$

with $0 \leq a_{\text {diff }}<1$. The following computations were performed with $R_{0}=$ $0.8 \mathrm{~nm}, R_{N} \approx 1.78 \mathrm{~nm}, u\left(R_{0}\right)=-14.39, n_{\mathrm{pot}}=4, \Gamma=0.2 \mathrm{~nm}, f_{\mathrm{pot}}=2$, $D(\infty)=6 \times 10^{9} \mathrm{~nm}^{2} \mathrm{~s}^{-1}$ and $a_{\text {diff }}=0.5$. The corresponding function $u(r)$ is shown in Fig. 7. The present values of $R_{0}, u\left(R_{0}\right)$, and $D(\infty)$ roughly correspond to the formation and dissociation of pyrene excimers in a fluid solvent like hexane at room temperature (cf. the Appendix).

Starting from $f=1.002, N=400$ and polynomials of the degree $2 J=4$, the numerical accuracy was increased either by a reduction of $f$ (and a corresponding increase of $N$ ) or by an increase of $J$. Two sets of computations were performed, whose main results are presented in Fig. 8. The computations of the first set had $2 J=4$ and $t_{\mathrm{a}} \approx 1.421 \times 10^{-23} \mathrm{~s}$ in common, and the values of $f$ and $N$ were varied: $f=1.002, N=400 ; f=1.002^{1 / 2}$ $\approx 1.001, N=800 ; f=1.002^{1 / 4} \approx 1.0005, N=1600$. The computations of the second set had $f=1.002, N=400$, and $t_{\mathrm{a}} \approx 3.638 \times 10^{-21} \mathrm{~s}$ in common, and the degree of the polynomials was varied: $2 J=4,6,8$. The final stationary distributions were independent of the value of $t_{\mathrm{a}} \cdot t_{\text {stat }}=512 \mathrm{~ns}$ was a practically infinitely long time: $w\left(r_{i}, t_{\text {stat }} \mid r_{N}\right)$ agreed with $w\left(r_{i}, t_{\text {stat }} \mid\right.$ $r_{1}$ ) with an accuracy of 13 digits. 


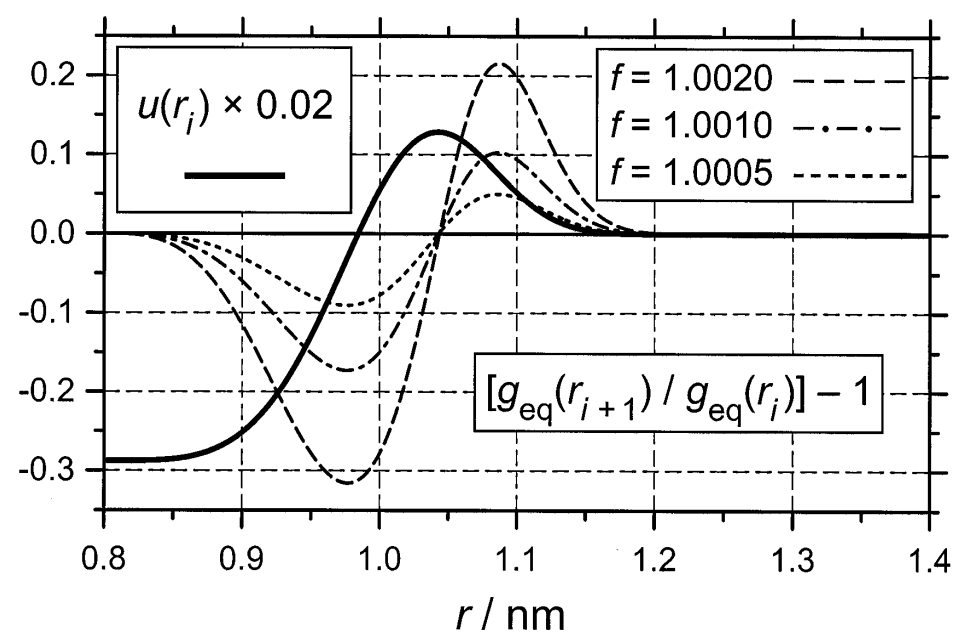

Fig. 7. Reduced potential $u(r)$, calculated with Eq. (79) and the parameters $R_{0}=0.8 \mathrm{~nm}$, $u\left(R_{0}\right)=-14.39, n_{\text {pot }}=4, \Gamma=0.2 \mathrm{~nm}$, and $f_{\text {pot }}=2$. The ratio of successive equilibrium values of $g, g_{\mathrm{eq}}\left(r_{i+1}\right) / g_{\mathrm{eq}}\left(r_{i}\right)=\exp \left(-\left[u\left(r_{i+1}\right)-u\left(r_{i}\right)\right]\right)$, was calculated with the radial increment factors $f=1.002,1.001$, and 1.0005 .

The increase of numerical accuracy by a reduction of $q=f-1$ is demonstrated in Fig. 8b. The almost constant systematic error $F$ for $r>1.05 \mathrm{~nm}$ ranges from $\approx 1.83 \times 10^{-5}$ for $q \approx 0.0005$ to $\approx 4.75 \times 10^{-3}$ for $q=0.002$ and is rather accurately proportional to $q^{4}$. The irregular systematic error $F_{1}$ due to the imperfect implementation of the boundary condition ranges from $\approx 5.1 \times 10^{-6}$ for $q \approx 0.0005$ to $\approx 1.99 \times 10^{-4}$ for $q=0.002$ and is rather accurately proportional to $q$.

In Fig. $8 \mathrm{c}$ the increase of numerical accuracy by the increase of the degree of the interpolation polynomials is demonstrated. In order to eliminate the irregularities due to the boundaries, the difference $\left(F_{390}-F_{11}\right)$ is taken as a measure for the attainable accuracy: $\left(F_{390}-F_{11}\right)_{J=2} \approx 4.75 \times$ $10^{-3},\left(F_{390}-F_{11}\right)_{J=3} \approx-1.36 \times 10^{-4},\left(F_{390}-F_{11}\right)_{J=4} \approx 3.72 \times 0^{-6}$. Thus, in the present example, an increase of the degree of the polynomials by 2 entails a reduction of the systematic error due to the potential to $\approx 1 / 36$.

\subsection{Example 5: $u=u(r), D=D(r)$, very large radial range with increasing ratio $r_{i+1} / r_{i}$}

The last example differs from the preceding one in two respects: First, $q=$ $f-1$ is gradually increased from $q_{1} \approx 0.002$ to the final value $q_{N} \approx 0.02$ (see Fig. 9b). Second, the radial range is extremely large: $R_{0}=0.8 \mathrm{~nm}$ and $R_{N} \approx 8 \times 10^{5} \mathrm{~nm}(N=1409)$. As a representative example, the evolution of the fundamental solution with $r_{n} \approx 800 \mathrm{~nm}(n=1043)$ is shown in 


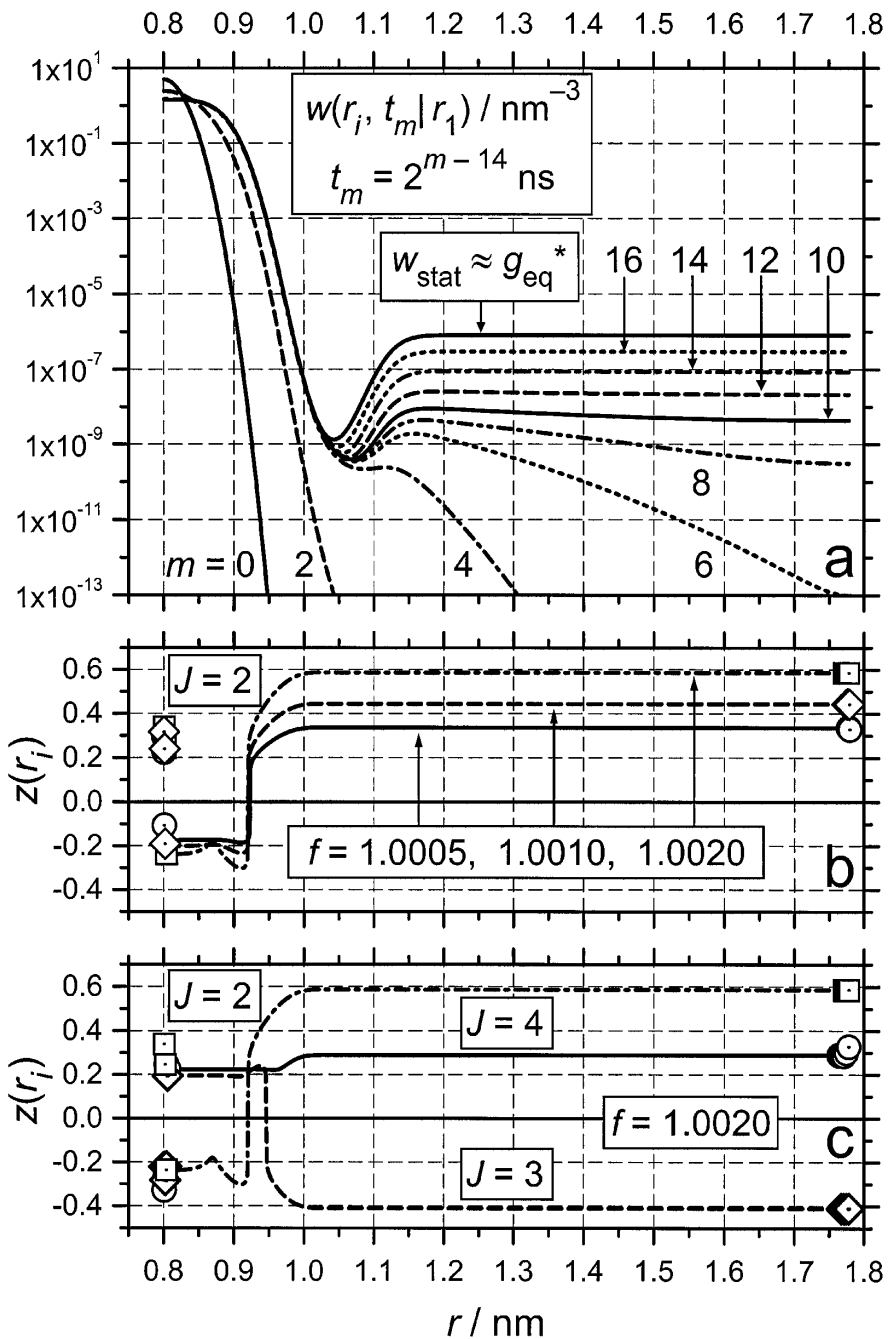

Fig. 8. Example 4: Relative diffusion in a small radial range with a reduced potential $u=u(r)$ (cf. Fig. 7), $D=D(r)\left(a_{\text {diff }}=0.5, D(\infty)=6 \times 10^{9} \mathrm{~nm}^{2} \mathrm{~s}^{-1}\right.$; cf. Eq. (80)) and reflecting boundaries at $R_{0}=0.8 \mathrm{~nm}$ and $R_{N} \approx 1.78 \mathrm{~nm}$. (a) $w$ was calculated with $f=$ $1.002, N=400, t_{\mathrm{a}} \approx 1.084 \times 10^{-28} \mathrm{~s}$, and $J=4$. The times $t_{m}=2^{m-14} \mathrm{~ns}$ for the curves in the figure range from $t_{0} \approx 61 \mathrm{fs}$ to $t_{16}=4 \mathrm{~ns}$. The solid curve with the label $\infty$ is the stationary distribution, which is reached at $t_{\text {stat }}=t_{23}=512 \mathrm{~ns}\left(w_{\text {stat }}\right.$ is independent of $r_{n}$ with an accuracy of 13 digits). (b) $J=2$ : Effect of radial increment factor $f$ on the relative deviation $z$ of $w_{\text {stat }}$ from the equilibrium distribution $g_{\text {eq }}^{*}(f=1.002, N=400$; $f=1.002^{1 / 2} \approx 1.001, N=800 ; f=1.002^{1 / 4} \approx 1.0005, N=1600$ ). The three leftmost and the three rightmost points of each $z$-curve are represented by separate symbols. (c) $f=1.002, N=400$ : Effect of the degree $2 J$ of the interpolation polynomials on the relative deviation $z$ of $w_{\text {stat }}$ from the equilibrium distribution $g_{\mathrm{eq}}^{*}$. The $J+1$ leftmost and $J+1$ rightmost points of each $z$-curve are represented by separate symbols. 


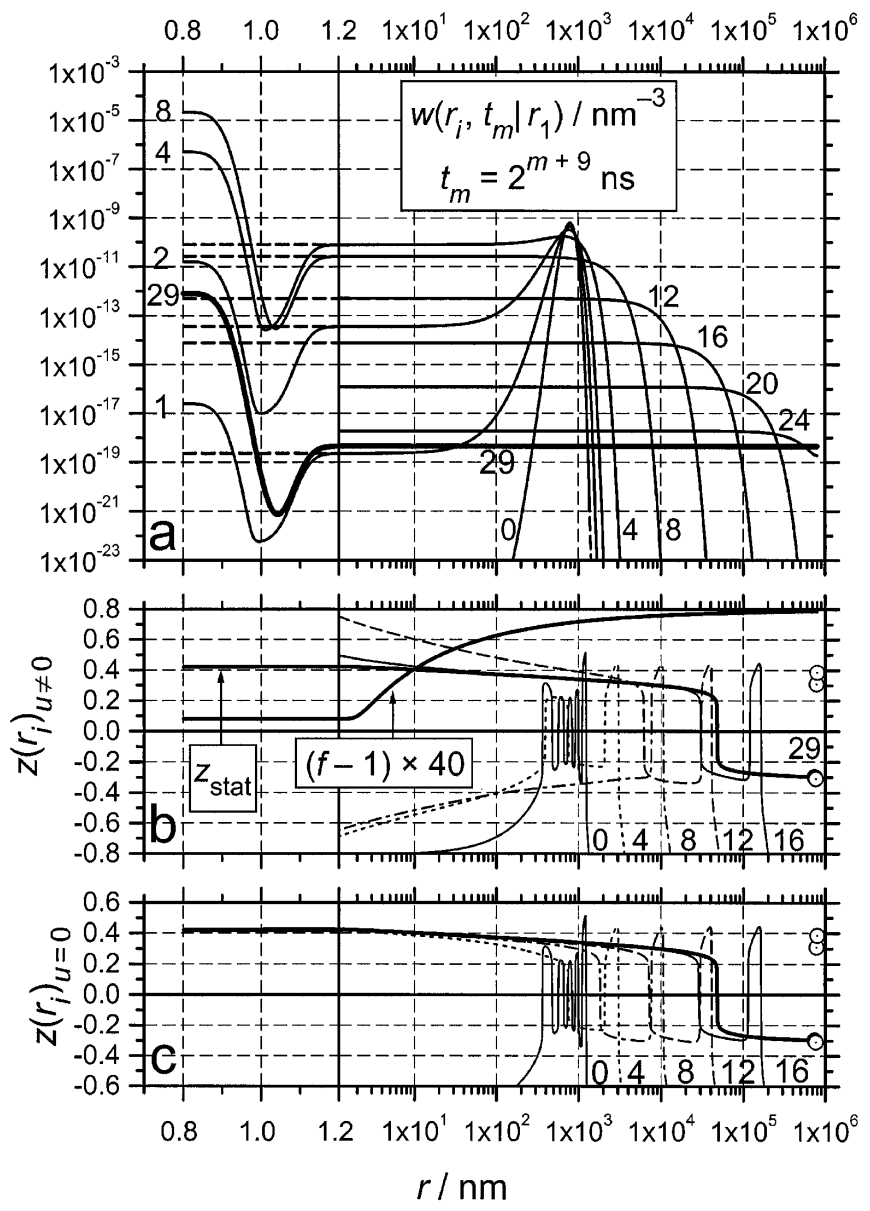

Fig. 9. Example 5: Relative diffusion with the same reduced potential $u(r)$ and relative diffusion coefficient as in Example 4 (Fig. 8), but with a very large radial range: $R_{0}=$ $0.8 \mathrm{~nm}$ and $R_{N} \approx 8 \times 10^{5} \mathrm{~nm}\left(f_{1}=1.002, f_{2}=1.02, N=1409\right.$, transition from $f_{1}$ to $f_{2}$ with Eq. (56) and $\kappa=16$ and $i_{1-2}=300, r_{1} \approx 0.8008 \mathrm{~nm}, r_{N} \approx 8.005 \times 10^{5} \mathrm{~nm}, t_{\mathrm{a}} \approx$ $\left.1.084 \times 10^{-28} \mathrm{~s}\right)$. All curves refer to a start position $r_{n} \approx 800 \mathrm{~nm}(n=1043)$. The abscissa is linear from 0.7 to $1.2 \mathrm{~nm}$ and logarithmic from 1.2 to $10^{6} \mathrm{~nm}$. (a) Numerical fundamental solutions $w$ (-): The times $t_{m}=2^{m+9} \mathrm{~ns}$ for the curves in the figure range from $t_{0}=512 \mathrm{~ns}$ to $t_{29}=t_{\text {stat }} \approx 275 \mathrm{~s}$ (thick solid curve, $m=29$ ). Since for times $t_{8}<t_{m}<$ $t_{24}$ the shape of the curves $w\left(r_{i}, t_{m} \mid r_{n}\right)$ remains virtually constant in the range $0.8 \mathrm{~nm}<$ $r_{i}<1.2 \mathrm{~nm}$ (only the amplitude decreases), these curves are not shown. For comparison the theoretical curves $g^{*}(---)$ were calculated for the times $t_{0}$ to $t_{16}$ with Eqs. (77) and (78), $D=D(\infty)$, and $u=0$. (b) Relative deviation $z$ of $w$ from $g^{*}\left(m \leq 16, r_{i}>1.2 \mathrm{~nm}\right.$, thin curves) and of $w_{\text {stat }}$ from the equilibrium distribution $g_{\text {eq }}^{*}$ (thick solid curve); the four rightmost points of $z_{\text {stat }}$ are represented by open circles. The curve $q=f-1$ was calculated with Eq. (56) and the parameter values given above. (c) Relative deviation $z$ of $w$ (computed also with $D=D(\infty)$ and $u=0$ ) from $g^{*}$; the thick solid curve is identical with curve 29 in Fig. 9 b. 
Fig. 9a. The scale for the radius is linear from 0.7 to $1.2 \mathrm{~nm}$ and logarithmic from 1.2 to $10^{6} \mathrm{~nm}$. Since the initial evolution takes place in the radial range where $u$ and $D$ are virtually constant, $w\left(r_{i}, t \mid r_{n}\right)$ can be compared with the theoretical curves $g^{*}\left(r_{i}, t \mid r_{n}\right)$, calculated with Eqs. (77) and (78) (the value of $\varepsilon$ ranged from $1.62 \times 10^{-4}$ with curve 0 to $1.84 \times 10^{-4}$ with curve 16 ). The main results are:

(a) The $N$ numerical fundamental solutions $w\left(r_{i}, t \mid r_{n}\right)$ were equal within 13 digits at the time $t_{\text {stat }}=t_{\mathrm{a}} \times 2^{101} \approx 2.749 \times 10^{2} \mathrm{~s}\left(t_{\mathrm{a}} \approx 1.084 \times 10^{-28} \mathrm{~s}\right)$. Thus the last time step $\left(\approx 1.374 \times 10^{2} \mathrm{~s}\right)$ was by 30 orders of magnitude longer than the first time step.

(b) The maximum relative error $F$ of about $1.8 \times 10^{-4}$ appears now in the near zone at $\approx 1 \mathrm{~nm}$. This is a consequence of the large radial range and the increasing factor $f$. Therefore, although the equilibrium probability density in the first shell is by a factor of $1.8 \times 10^{6}$ greater than that in the $N$-th shell, the equilibrium probability $V_{N} g_{\text {eq }}\left(r_{N}\right) \approx 5.67 \times 10^{-2}$ is about $5.5 \times 10^{12}$ times larger than the equilibrium probability $V_{1} g_{\mathrm{eq}}\left(r_{1}\right) \approx$ $1.04 \times 10^{-14}$

(c) For large radii, where the potential $u$ and the relative diffusion coefficient $D$ are virtually constant, the relative deviation $F$ of $w$ from the theoretical curves $g^{*}$ is small (see Fig. 9b).

(d) The analogous computations were performed with constant potential $u$ and constant relative diffusion coefficient $D=D(\infty)$. The corresponding relative deviations $z$ of $w$ from the theoretical curves $g^{*}$ are shown in Fig. 9c. Between 0.8 and $2 \times 10^{4} \mathrm{~nm}$, curve 16 in Fig. $9 \mathrm{c}$ virtually coincides with curve $z_{\text {stat }}$ of Fig. $9 \mathrm{~b}$. That means, if $q$ is virtually constant in the near zone and the degree of the polynomials is high enough as in this example, the contribution of the near zone to the total systematic error remains small.

\subsection{Implementation of reactions}

The effect of a distance-dependent rate coefficient $k$ on a distribution $\rho\left(r_{i}, t\right)$ can be taken into account in different ways. The simplest way is its implementation in the fundamental solutions. Let $1 / k$ be in the range $\tau_{\min } \leq 1 / k \leq \tau_{\max }$ and let the times $t_{\mathrm{b}}, t_{\mathrm{c}}, t_{\mathrm{d}}$ satisfy the relation

$$
t_{\mathrm{a}} \leq t_{\mathrm{b}} \leq t_{\mathrm{c}} \ll \tau_{\min } \leq \tau_{\max }<t_{\mathrm{d}} \approx 7 \tau_{\max } .
$$

$t_{\mathrm{b}}$ is the time at which the reaction is taken into account for the first time. $t_{\mathrm{c}}$ is the adequate time step for calculating the course of the reaction, and $t_{\mathrm{d}}$ is the longest time of interest for the given kinetic problem. If $w\left(r_{i}, t_{\mathrm{b}} \mid r_{n}\right)_{\mathrm{diff}}$ is a fundamental solution implying pure diffusion, then the corresponding fundamental solution implying also reaction is given by

$$
w\left(r_{i}, t_{\mathrm{b}} \mid r_{n}\right)_{\text {react }} \approx w\left(r_{i}, t_{\mathrm{b}} \mid r_{n}\right)_{\text {diff }} \times \exp \left[-k\left(r_{i}\right) t_{\mathrm{b}}\right] .
$$


The further evolution of the fundamental solutions is calculated as before up to the time $t_{\mathrm{c}}$. Finally, when the time $t_{\mathrm{c}} \approx 0.01 \tau_{\min }$ is reached, the evolution of the distribution function $\rho$ is calculated with procedure (III) with constant time steps $t_{\mathrm{c}}$ up to a time $t_{\mathrm{d}}$ (cf. section 2.6):

$$
\rho\left(r_{i},(v+1) \times t_{\mathrm{c}}\right)=\sum_{n=1}^{N}\left[\rho\left(r_{n}, v t_{\mathrm{c}}\right) \times V_{n}\right] \times w_{n}\left(r_{i}, t_{\mathrm{c}} \mid r_{n}\right) .
$$

Note that the computation time needed for each time step of length $t_{\mathrm{c}}$ is only $1 / N$ of the time needed for computing the $N$ fundamental solutions in a time-doubling cycle (if all elements of all fundamental solutions significantly differ from zero).

Within the frame of double-precision computations, the practical choice of $t_{\mathrm{b}}$ is governed by two conditions. First, $t_{\mathrm{b}} / \tau_{\min }$ should be very small, and in this respect $t_{\mathrm{b}}=t_{\mathrm{a}}$ would be the best choice, if the effect of round-off errors is neglected. Second, $t_{\mathrm{b}} / \tau_{\max }$ must be large enough, e.g. $t_{\mathrm{b}} / \tau_{\max } \geq 10^{-10}$, in order that the round-off error in the computation of $w\left(r_{i}, t_{\mathrm{b}} \mid r_{n}\right)_{\text {react }}$ remain small enough for arbitrary $r_{i}$. Obviously both conditions can be simultaneously satisfied as long as the ratio $\tau_{\max } / \tau_{\min }$ is not extremely large (e.g. $\leq 10^{\circ}$ ).

\subsection{Computational aspects}

The numerical calculations were performed on a personal computer with $128 \mathrm{MB}$ random-access memory and a $366 \mathrm{MHz}$ Intel Pentium II processor. The computer programs were written in FORTRAN 90 (compiler: Absoft Pro Fortran, version 6.2). The computer memory requirements are almost completely given by two two-dimensional arrays of $N^{2}$ double-precision floating-point numbers for the storage of the $N$ fundamental solutions and a buffer for the intermediate storage of a new set of them. Thus, for $N=$ 1000 , roughly $16 \mathrm{MB}$ of random-access memory are required. The length of the executable programs was kept below $1 \mathrm{MB}$ by defining most large arrays as allocatable arrays. No special efforts were made to optimize the computer programs. With $N=1000$, one cycle of time-doubling took $t_{\text {cycle }}$ $\approx 4$ min, when all $N$ elements of all $N$ fundamental solutions were significantly different from zero.

The interpolation polynomials were calculated with Newton's method $[21,22]$, which yields the polynomials in a form that is very suitable for calculating their first and second derivatives. Most of the mathematical operations can be formulated as matrix operations. From a practical point of view the present use of explicit summations seems to be preferable, because it is better suited for limiting all summations to those terms that are significantly different from zero. At least in the initial time-doubling cycles, the speed of the computation of $w\left(r_{i}, t \mid r_{n}\right)$ is enhanced by several orders of 
magnitude, if all extremely small elements $w\left(r_{i}, t \mid r_{n}\right)$ are equated with zero and only the remaining elements are taken into account. The computation of the curves in Fig. 8a may serve as an example $\left(N=1600\right.$ and $t_{\text {cycle }} \approx$ $16 \mathrm{~min})$. The initial speed of computation was strongly enhanced by equating all $w\left(r_{i}, t \mid r_{n}\right)<10^{-50} g_{\text {eq }}\left(r_{i}\right)$ with zero and by limiting all summations to terms that differed from zero. Curve 2 in Fig. $8 \mathrm{a}$ is the last one $(t \approx$ $244 \mathrm{fs})$, in which part of the values $w\left(r_{i}, t \mid r_{1}\right)$ are equal to zero. The 56 cycles needed for computing curve 2 required $38 \mathrm{~min}$. The remaining 21 cycles for computing the stationary distribution $\left(t_{\text {stat }}=512 \mathrm{~ns}\right)$ required $340 \mathrm{~min}$

\section{Discussion}

\subsection{Merits and deficiencies of the algorithm}

The present algorithm was criticized in several respects. Part of the criticism has been already answered in the Introduction and by the numerical solution of the Ornstein-Uhlenbeck problem in section 2.7. Some other points of the criticism, however, deserve an explicit discussion and are treated in the following items.

Comparison with other algorithms. One may ask whether the present algorithm permits one to solve diffusion problems of interest with higher accuracy and in shorter time than any other known algorithm. This question cannot be definitively answered by the author for two reasons. First, the algorithms of most interest [16-19] are mathematically complex, and their implementation would have been difficult for the author. Moreover, the published details are not always sufficient for writing the pertinent program code $[16,19]$. Second, although general statements on the accuracy of algorithms can be found in the literature, there is a lack of illustrative examples that demonstrate the range of application and achievable accuracy of a particular algorithm. For these reasons an alternative procedure is suggested. A reader may apply the algorithms at his disposal to the diffusion problems in examples 1 to 5 . If he will be able to surpass the present results with respect to numerical accuracy and speed of computation, then the present algorithm will be of no interest to him. To facilitate this comparison, in particular the relative deviations of numerical solutions from exact solutions are shown in a suitable form.

Time-doubling. The repeated time-doubling in the present algorithm may look similar to the doubling-time in the boundary-doubling method developed by Kim et al. [20]. The meaning of the term doubling-time is, however, completely different from the meaning of the term time-doubling in the present algorithm. The doubling-time in ref. [20] is the time at which the outer boundary is doubled. If simultaneously the radial step width $\Delta r$ is 
doubled, then the time step $\Delta t$ can be quadrupled (adaptive step size control by step doubling). After each adaptation of step size, however, $\Delta r$ and $\Delta t$ remain constant for many successive steps.

Interpolating functions. In certain diffusion or heat-conduction problems, an exponential approximation closely corresponds to the local concentration or temperature profile in a space layer and seems to give the best results [34]. For this reason it was argued that a polynomial interpolation cannot be advantageous for the present purpose. However, close to minima or maxima of a distribution, an exponential approximation can be no longer optimal and is likely to be inferior to the present polynomial approximation, apart from the loss of mathematical simplicity.

Degree of the polynomials. The very small relative error over many orders of magnitude of the numerical fundamental solutions $w$ is perhaps the most surprising result of the present investigation and not yet completely understood. The strong increase of numerical accuracy with increasing degree of the polynomials can be easily understood (cf. pp. 198-200 in ref. [21]). One may ask which gain in accuracy is expected by using polynomials of higher degree than 8. Probably, within the limitations of doubleprecision calculations, it will not be possible to calculate the required first and second derivatives of the polynomials with sufficient accuracy, if the polynomials are calculated with Newton's algorithm [21] as in the present computer program. The calculation of the data in Table 1 revealed that the smallest terms in a column are sums of large terms with alternating signs. In conclusion, within the limitations of double-precision accuracy, polynomials of the $8^{\text {th }}$ degree seem to be an optimum. Finally it should be noted that the total computation time is virtually independent of the degree of the polynomials.

Boundaries. Another limitation of the attainable accuracy results from the present implementation of a reflecting or absorbing boundary. The systematic errors resulting from this imperfect implementation are not reduced by increasing the degree of polynomials. One might expect that these systematic errors are smaller, when $(\Delta r)_{i}=r-r_{i-1}$ is constant near the boundaries. In this connection a smooth transition from $(\Delta r)_{2}$ to $(\Delta r)_{N}$ with $(\Delta r)_{2} \ll(\Delta r)_{N}$ has been of interest. The pertinent computations yielded, however, no significant reduction of the systematic error.

Detailed balance. Nadler and Schulten [15] have shown that the approximate calculation of partial derivatives with a finite-difference method guarantees neither probability conservation nor a detailed balance. They and some of their successors $[16,19]$ emphasized the importance of a detailed balance for a good diffusion algorithm. The author has not been able to share this view. On the contrary, it seems to him more likely that, by implementing a detailed balance, in general one cannot obtain an optimal algorithm for the evolution of a distribution function. Apart from the illustration of this statement in section 2.8 and Fig. 4, the following consider- 
ation may be helpful. The numerical solution of a pure diffusion problem can be compared with the fitting of a function to a set of data points. Equating the stationary distribution with the equilibrium distribution by the enforced detailed balance corresponds to forcing the fitted function to pass exactly through one of the data points. In general the fitted function thus obtained will not be the optimal function for the whole data set. In the case of a detailed balance, the equality of the stationary distribution with the equilibrium distribution is only a necessary condition for a correct program code, but contains no information on the quality of the algorithm. Without an exact detailed balance, the deviation of the stationary distribution from the equilibrium distribution already contains information on the quality of the algorithm.

Nonnegativity. Physically, negative probability densities have no meaning and should not occur in a good diffusion algorithm that involves only transfer of probability density to adjacent grid points. This is also true for the present algorithm with polynomials of the second degree. With higher polynomials $(J \geq 4)$, negative probability densities occur at the relative positions $j= \pm 2, \pm 4, \ldots$ (see Table 1 ). In the actual calculation of the numerical fundamental solutions $w$, negative values occur only in those ranges of $x$ or $r$, where exact values $g_{i}$ and $g_{i+1}$ differ by an order of magnitude or more.

Restriction to first order with respect to time. The restriction to first order with respect to time would indeed be a disadvantage of an algorithm working with constant time steps $t_{\mathrm{a}}$. The distinctive feature of the present algorithm is, however, that the time needed for the computation of $\rho\left(r_{i}, t\right)$ in the time range of interest is almost independent of the length $t_{\mathrm{a}}$ of the first time step. Hence $t_{\mathrm{a}}$ can be chosen almost arbitrarily short, and nothing would be gained by a second-order procedure with respect to time.

Stability of first-order scheme. The fact that in general an extremely short first time step $t_{\mathrm{a}}$ has been used was misinterpreted as evidence for a numerical instability of the algorithm in the case of larger values of $t_{\mathrm{a}}$. The opposite is true, as is illustrated by example 1 and Fig. 1: In the absence of reactions, the stationary numerical distribution $w_{\text {stat }}$ is independent of $t_{\mathrm{a}}$ as long as $t_{\mathrm{a}}$ is below the sharp stability limit. The unavoidable accumulation of round-off errors is not a peculiarity of the present algorithm but results from the limitations imposed by double-precision accuracy (cf. e.g. section 1.3.4 in ref. [24]).

Renormalization of the numerical fundamental solutions. Any numerical procedure will in the end be limited by the accumulation of round-off errors. This limitation will be the more obvious the more accurate the given procedure is. Moreover, example 3 in section 3.4 shows that even without renormalization a very high numerical accuracy is achieved. An unavoidable numerical error results from the combination of a large radial range with a very short first time step $t_{\mathrm{a}}$. In example 5, for instance, $w\left(r_{N}, t \mid r_{N}\right)$ 
remains constant in the whole time range from $t=0$ to $t=t_{\mathrm{a}} \times 2^{39} \approx$ $2.98 \times 10^{-17} \mathrm{~s}$, whereas the ratio $w\left(r_{N-1}, t \mid r_{N}\right) / w\left(r_{N}, t \mid r_{N}\right)$ steadily increases from 0 to $\approx 10^{-15}$ in the same time range.

Renormalization of the theoretical fundamental solutions. The present use of renormalized theoretical fundamental solutions $g^{*}$ is consistent with the normalization of the theoretical equilibrium distributions, which is tacitly applied in other algorithms.

Corrections. The correction of the quantities $H_{n, j}$ (see Eq. (67)) according to Eq. (73) is to some degree arbitrary and therefore unsatisfactory. However, this correction can easily be kept very small, and it is completely negligible in comparison to the large systematic errors that would be introduced by using the recommended detailed balance [15, 16, 19]. Moreover, the very weak dependence of the numerical fundamental solution on the particular correction of the quantities $H_{n}$ shows that the correction for probability conservation is not a limiting factor in the application of the algorithm (as long as this correction is very small).

Control parameters. An ideal algorithm should contain control parameters that limit the maximum deviation of the numerical solution from the true solution of a partial differential equation. Admittedly, for the present algorithm no control parameters are given. However, this should not seriously limit its usefulness. The practical application of the algorithm to threedimensional diffusion is governed by a few rules:

Rule 1. If possible, a constant radial increment factor $f=1+q$ with $q \lesssim 0.02$ should be used.

Rule 2. The maximum acceptable value of $q$ is obtained from the limits $\sigma^{-1}$ and $\sigma$ for the acceptable ratio of neighboring equilibrium values $\rho_{\text {eq }}$, (cf. Eq. (3)).

Rule 3. The ratio $\sigma$ can be the greater the higher the degree $2 J$ of the interpolation polynomials. For this reason, $2 J=8$ will be in general the best choice.

Rule 4. If a variable radial increment factor $f_{i}=1+q_{i}$ is needed, the best results are obtained, if $q$ is virtually constant in the effective range of the potential. The relative error $\omega_{n}$ in the probability conservation during the first time step (cf. Eq. (70)) should be extremely small in the whole radial range, e.g. $\left|\omega_{n}\right|<10^{-8}$.

Rule 5. In the case of reflecting boundaries, the first time step $t_{\mathrm{a}}$ can be made extremely short, for instance by a factor $10^{-12}$ shorter than the maximum $\left(t_{\mathrm{a}}\right)_{\max }$ corresponding to the limit of numerical stability. The concomitant accumulation of round-off errors is corrected for by a renormalization of all $N$ fundamental solutions after every ten time-doubling cycles up the diffusion time $t_{\mathrm{b}}$, when the reaction term $k(r)$ is taken into account (cf. section 3.7).

Rule 6. In the case of absorbing or constant-concentration boundaries, the accumulation of round-off errors can no longer be corrected for by a 
renormalization. Therefore the first time step $t_{\mathrm{a}}$ should not be made extremely short, but still much shorter than $\left(t_{2}\right)_{\max }$.

Rule 7. The convergence of the algorithm with decreasing $q$ should be checked.

Waste of computer resources. This objection would have been justified in 1980, when the present computations would have required a large computer, shared by a community of researchers. At the end of the year 1999, when the typical random-access memory of a new personal computer was $64 \mathrm{MB}$, this objection did not make sense anymore.

Soft-sphere approximations. The relative ease, with which the combination of a short-range potential with a large radial range can be treated, offers the possibility to overcome the limitations of hard-sphere models. A suitable repulsive potential is introduced, and the inner reflecting boundary is shifted to a smaller radius, where the repulsive term of the potential already strongly dominates (and where the equilibrium probability density is already much smaller than in the potential minimum). Example 1 in section 2.7 has shown that the present implementation of a reflecting boundary yields acceptable results even if the potential does not satisfy Eq. (35).

\subsection{On the most efficient algorithm for long time propagations}

The alleged slowness of the present algorithm was perhaps the main objection against it. The argument is simple: If only one distribution function at a single time $t$ is needed, it is an enormous waste of computation time to compute $N$ distribution functions instead of one. In practice, however, nearly always the evolution of a distribution function is of interest and, for instance, 100 to 1000 distributions with constant time steps $t_{\mathrm{c}}$ are needed (cf. section 3.7).

The computation time needed for a time-doubling cycle, $t_{\text {cycle }}$, is proportional to the third power of the number of the fundamental solutions, $N$, if all elements of each fundamental solution significantly differ from zero. Obviously, due to this fact, the present algorithm is very slow if $N$ is large. For instance, with $N=1600$ (cf. section 3.8) and without parallel computing, it would be difficult to fit the parameters of a kinetic model to experimental data within a reasonable time. However, in many cases, values of $N$ in the range $100 \lessgtr N \lesssim 200$ will be sufficient and the time $t_{\mathrm{d}}$, up to which the distribution $\rho\left(r_{i}, t\right)$ is to be calculated, may be much shorter than the time needed for complete equilibration in the absence of a reaction. In such cases the time for the computation of the whole evolution of $\rho$ reduces to about $1 \mathrm{~min}$ or less, and the present algorithm becomes applicable as part of a curve-fitting procedure.

In a comparison of different algorithms that allow the application of very large constant time steps $t_{\mathrm{c}}$, the distinction between two different computation times is important. The first computation time is the time needed 
for computing $\rho\left(r_{i}, t_{\mathrm{c}}\right)$, which requires in the present algorithm the computation of $N$ fundamental solutions $w\left(r_{i}, t_{\mathrm{c}} \mid r_{n}\right)$. The second computation time is the time needed for computing $v_{\mathrm{d}}$ distribution functions $\rho\left(r_{i}, t_{\mathrm{c}} v\right)$ with $v=$ $1,2, \ldots, v_{\mathrm{d}}$ and $t_{\mathrm{d}}=t_{\mathrm{c}} v_{\mathrm{d}}$. With respect to the second computation time, the present algorithm seems to the most efficient one, because there is no simpler solution for this problem than the summation of $N$ weighted fundamental solutions. The first computation time will be in general longer than the second computation time. For instance, if $v_{\mathrm{d}}=N$ and $w\left(r_{i}, t_{\mathrm{c}} \mid r_{n}\right) \neq 0$ for arbitrary $n$ and $i$, then the time needed for computing the $v_{\mathrm{d}}$ distribution functions $\rho\left(r_{i}, t_{\mathrm{c}} v\right)$ would be equal to be the time needed for computing a new set of $N$ fundamental solutions $w\left(r_{i}, 2 t_{\mathrm{c}} \mid r_{n}\right)$.

With the algorithm in refs. $[16,19]$ the situation is reversed. Due to the direct computation of the distribution $\rho\left(r_{i}, t_{\mathrm{c}}\right)$, the first computation time can be rather short. On the other hand, the same procedure is to be repeated $\left(v_{\mathrm{d}}-1\right)$ times; hence, even in the most favorable case, the second computation time will be at least $\left(v_{\mathrm{d}}-1\right)$ times longer than the first computations time. An advantage of that algorithm is that the time step need not be constant.

It is not a priori evident, which of the two algorithms is more efficient in computing the evolution of a distribution function. Finally, if for some problems of interest the present algorithm will turn out to be the only practicable one, slowness will be no longer an argument.

\section{Summary}

With the present algorithm, the Smoluchowski equation of a spherically symmetric three-dimensional diffusion problem can be numerically solved with high accuracy even in the unfavorable case of a short-range potential barrier in combination with a large radial range. The basic features of the algorithm are:

(a) A series of $N$ spherical shells of volume $V_{i}$ is defined by the radii $R_{0}, R_{1}, \ldots, R_{i}, \ldots, R_{N}$ with $R_{i+1} / R_{i}=1+q$, where $q$ is either constant or a slowly increasing function of $i$. The mean radii $r_{i}$ of the spherical shells are taken as grid points.

(b) Numerical fundamental solutions $w\left(r_{i}, t \mid r_{n}\right)$ are defined. $w\left(r_{i}, 0 \mid\right.$ $\left.r_{n}\right)=V_{n}^{-1} \delta_{i n}$ is the numerical analogue of a $\delta$-function. All that is needed for the calculation of $w\left(r_{i}, t \mid r_{n}\right)$ is the set of fundamental solutions $w\left(r_{i}, t_{\mathrm{a}} \mid\right.$ $r_{n}$ ), where $t_{\mathrm{a}}$ is the duration of the first diffusion step.

(c) The first and second derivatives of the numeric $\delta$-function $w\left(r_{i}, 0 \mid\right.$ $r_{n}$ ) with respect to $r$ at $r_{i}$ are defined by the respective derivative of a polynomial of the degree $2 J(J=1,2,3,4)$, centered at $r_{i}$. In the calculation of $w\left(r_{i}, t_{\mathrm{a}} \mid r_{n}\right)$, the error in probability conservation becomes very small in the range of $q$ that is of practical interest $(q \lesssim 0.02)$. For very small $q$, the probability error is approximately proportional to $q^{2 J+2}$. 
(d) A reflecting inner boundary is implemented by compensating the loss due to diffusion from the shells $1,2, \ldots, J$ through the boundary by the gain due to the opposite diffusion from the auxiliary shells $0,-1, \ldots$, $-J+1$ through the boundary. An absorbing inner boundary is implemented by the same terms, but with opposite signs. Radiation and constant-concentration boundaries are implemented in a similar way. The treatment of outer boundaries is analogous.

(e) By successive doubling of the total diffusion time $t$, each fundamental function $w\left(r_{i}, 2 t \mid r_{n}\right)$ can be expressed as the weighted sum of all $N$ fundamental solutions $w\left(r_{i}, t \mid r_{l}\right)(l=1,2, \ldots, N)$. Since the total computation time is roughly proportional to the logarithm of the total diffusion time, the stationary distribution $w_{\text {stat }}$ can be always computed (in the case reflecting boundaries and in the absence of reactions).

(f) The evolution of an arbitrary distribution $\rho$ can be calculated, starting from an initial condition $\rho\left(r_{i}, 0\right)$, in time steps of the length $t_{\mathrm{c}}$ by repeatedly applying the set of fundamental solutions $w\left(r_{i}, t_{\mathrm{c}} \mid r_{l}\right)$.

(g) A distance-depending rate coefficient $k$ can be taken into account by multiplying at a time $t_{2} \ll k_{\max }^{-1}$ all $N^{2}$ values $w\left(r_{i}, t_{2} \mid r_{n}\right)$ with $\exp \left[-k\left(r_{i}\right) t_{2}\right]$.

(h) Within the limits of double-precision computations, the best results were obtained with polynomials of the degree 8 (the highest degree tested).

(i) The exact equality of the stationary distribution with the equilibrium distribution (enforced by a detailed balance) will in general not lead to the best approximation of the evolution of a distribution.

(j) With increasing $q_{i}\left(q_{N} \approx 10 q_{1}\right)$, a radial range of six orders of magnitude can be covered with a maximum relative error of $w$ of less than $2 \times 10^{-4}$

\section{Acknowledgements}

The author thanks Professor J. Troe for support of this work and Dr. W. Naumann for long discussions on diffusion-influenced reactions and for the theoretical treatment of some problems in the kinetics of triplet-triplet annihilation. The author also thanks Professor N. Ernsting, Dr. G. Käb, and Professor R. Schinke for explaining to him the Chebyshev time propagation. Finally, the detailed comments by a referee were helpful in writing the final version of this paper.

\section{Appendix}

The need of a new algorithm appeared in connection with a well-known special case of reversible excimer formation [10, 35-37]: The lowest excited singlet state ${ }^{1} \mathrm{~A}^{*}$ of an aromatic hydrocarbon $\mathrm{A}$ is populated by triplet- 
triplet annihilation ${ }^{3} \mathrm{~A}^{*}+{ }^{3} \mathrm{~A}^{*} \rightarrow{ }^{1} \mathrm{~A}^{*}+{ }^{1} \mathrm{~A}$. The subsequent reversible formation of excimers, ${ }^{1} \mathrm{~A}^{*}+{ }^{1} \mathrm{~A} \rightleftharpoons{ }^{1}(\mathrm{AA})^{*}$, and the observable intensity ratio of delayed monomer fluorescence and delayed excimer fluorescence depend on the initial spatial distribution $\rho(r, 0)$ of ${ }^{1} \mathrm{~A} *$ relative to ${ }^{1} \mathrm{~A}$, on an effective potential $U(r)$ for the attraction between ${ }^{1} \mathrm{~A}^{*}$ and ${ }^{1} \mathrm{~A}$, and on the rate constants for the radiative and nonradiative decays of ${ }^{1} \mathrm{~A}^{*}$ and ${ }^{1}(\mathrm{AA}) *$ to the respective ground state or triplet state. In the case of pyrene (the classic example of delayed excimer fluorescence [35]), the numerical solution of the corresponding Smoluchowski Eq. (1) has to satisfy the following requirements:

(a) $U(r)$ is a short-range potential. Hence the radial steps $\Delta r$ must be small, e.g. $\Delta r \approx 10^{-3} R_{0}$ or less, where $R_{0}$ is the contact distance between $\mathrm{A}$ and $\mathrm{B}$.

(b) The radial range is large with a maximum $r_{N} \approx 100 R$.

(c) The potential minimum is $U\left(R_{0}\right) \approx-h c \times 3000 \mathrm{~cm}^{-1}$.

(d) The temperature range of interest $\left(T_{\max } \approx 300 \mathrm{~K}, T_{\min } \approx 120 \mathrm{~K}\right)$ corresponds to the range $14 \lesssim|U(R)| / k_{\mathrm{B}} T \lesssim 36$.

(e) The relative diffusion coefficient $D$ ranges from $\approx 5 \times 10^{-8} \mathrm{~cm}^{2} \mathrm{~s}^{-1}$ at the lowest temperature to $\approx 5 \times 10^{-5} \mathrm{~cm}^{2} \mathrm{~s}^{-1}$ at the highest temperature.

(f) The constant time step is $\leqslant 10^{-16} \mathrm{~s}$; the longest time of interest is $\approx 5 \times 10^{-6} \mathrm{~s}$.

(g) The deviation of the numerical solution from the exact solution of Eq. (1) must be less than $1 \%$ in the time range of interest for all temperatures.

\section{References}

1. M. v. Smoluchowski, Physik. Zeitschr. 17 (1916) 585 [p. 588, Eq. (50)].

2. M. v. Smoluchowski, Z. Physik. Chem. 92 (1917) 129.

3. P. Debye, Transact. Electrochem. Soc. 82 (1942) 265.

4. S. A. Rice, Diffusion-Limited Reactions, vol. 25 of the series Comprehensive Chemical Kinetics, ed. C. H. Bamford, C. F. H. Tipper and R. G. Compton (Elsevier, Amsterdam, 1985).

5. A. A. Ovchinikov, S. F. Timashev and A. A. Belyi, Kinetics of Diffusion-Controlled Chemical Processes (Nova, Commack, 1989; Russian original: Moscow, Khimiya, 1986).

6. J. B. Pedersen and J. H. Freed, J. Chem. Phys. 58 (1973) 2746.

7. J. B. Pedersen and J. H. Freed, J. Chem. Phys. 59 (1973) 2869

8. Z. Schulten and K. Schulten, J. Chem. Phys. 66 (1977) 4616.

9. M. J. Pilling and S. A. Rice, J. Chem. Soc. Faraday Trans. II, 72 (1976) 792.

10. P. R. Butler and M. J. Pilling, J. Chem. Soc. Faraday II, 73 (1977) 886.

11. S. H. Northrup and J. T. Hynes, J. Chem. Phys. 69 (1978) 5246.

12. S. H. Northrup and J. T. Hynes, J. Chem. Phys. 71 (1979) 871.

13. G. Lamm and K. Schulten, J. Chem. Phys. 78 (1983) 2713.

14. P. Clifford, N. J. B. Green and M. J. Pilling, J. Phys. Chem. 88 (1984) 4171.

15. W. Nadler and K. Schulten, J. Chem. Phys. 84 (1986) 4015.

16. E. Pines, D. Huppert and N. Agmon, J. Chem. Phys. 88 (1988) 5620.

17. J. B. Pedersen, L. I. Lolle and J. S. Jørgensen, Chem. Phys. 165 (1992) 339. 
18. K. L. Aminov and J. B. Pedersen, Chem. Phys. 193 (1995) 297.

19. E. B. Krissinel' and N. Agmon, J. Comput. Chem. 17 (1996) 1085

20. H. Kim, S. Shin and K. J. Shin, J. Chem. Phys. 108 (1998) 5861.

21. R. Zurmühl, Praktische Mathematik für Ingenieure und Physiker, $4^{\text {th }}$ Edition (Springer-Verlag, Berlin, 1963).

22. L. F. Shampine and M. K. Gordon, Computer solution of ordinary differential equations - The initial value problem (W. H. Freeman, San Francisco, 1975), chapter 2.

23. W. H. Press, B. P. Flannery, S. A. Teukolsky and W. T. Vetterling, Numerical Recipes - The Art of Scientific Computing (FORTRAN Version) (Cambridge University Press, Cambridge, 1989).

24. C. W. Gear, Numerical initial values problems in ordinary differential equations (Prentice-Hall, Englewood Cliffs, 1971).

25. A. R. Mitchell and D. F. Griffiths, The finite difference method in partial differential equations (J. Wiley \& Sons, Chichester, 1980).

26. K. Dekker and J. G. Verwer, Stability of Runge-Kutta methods for stiff nonlinear differential equations (North-Holland, Amsterdam, 1984), chapter 10.

27. G. E. Uhlenbeck and L. S. Ornstein, Phys. Rev. 36 (1930) 823.

28. H. Tal-Ezer and R. Kosloff, J. Chem. Phys. 81 (1984) 3967.

29. C. Leforestier, R. H. Bisseling, C. Cerjan, M. D. Feit, R. Friesner, A.Guldberg, A. Hammerich, G. Jolicard, W. Karlein, H.-D. Meyer, N. Lipkin, O. Roncero and R. Kosloff, J. Comput. Phys. 94 (1991) 59.

30. J. Broeckhove and L. Lathouwers (Editors), Time-dependent quantum molecular dynamics (Plenum Press, New York, 1992).

31. S. Chandrasekhar, Rev. Mod. Phys. 15 (1943) 1.

32. H. A. Kramers, Physica 7 (1940) 284.

33. K. Razi Naqvi, K. J. Mork and S. Waldenstrøm, J. Phys. Chem. 84 (1980) 1315.

34. S. V. Patankar, Numerical heat transfer and fluid flow (Taylor \& Francis, 1980).

35. C. A. Parker and C. G. Hatchard, Trans. Faraday Soc. 59 (1963) 284

36. J. B. Birks, B. N. Srinivasan and P. S. McGlynn, J. Mol. Spectr. 27 (1968) 266.

37. B. Stevens and M. I. Ban, Mol. Cryst. 4 (1968) 173. 